\title{
Organic molecules in protoplanetary disks around T Tauri and Herbig Ae stars ${ }^{\star}$
}

\author{
W.-F. Thi ${ }^{1,2,3}$, G.-J. van Zadelhoff ${ }^{1,4}$, and E. F. van Dishoeck ${ }^{1}$ \\ ${ }^{1}$ Leiden Observatory, PO Box 9513, 2300 RA Leiden, The Netherlands \\ e-mail: ewine@strw.leidenuniv.nl \\ 2 Department of Physics and Astronomy, University College London, Gower Street, London WC1E 6BT, UK \\ 3 Sterrenkundig Instituut Anton Pannekoek, Kruislaan 403, 1098 SJ Amsterdam, The Netherlands \\ ${ }^{4}$ Koninklijk Nederlands Meteorologisch Instituut, PO Box 201, 3730 AE De Bilt, The Netherlands
}

Received 14 January 2003 / Accepted 15 June 2004

\begin{abstract}
The results of single-dish observations of low- and high- $J$ transitions of selected molecules from protoplanetary disks around two T Tauri stars (LkCa 15 and TW Hya) and two Herbig Ae stars (HD 163296 and MWC 480) are reported. Simple molecules such as $\mathrm{CO},{ }^{13} \mathrm{CO}, \mathrm{HCO}^{+}, \mathrm{CN}$ and $\mathrm{HCN}$ are detected. Several lines of $\mathrm{H}_{2} \mathrm{CO}$ are found toward the T Tauri star LkCa 15 but not in other objects. No $\mathrm{CH}_{3} \mathrm{OH}$ has been detected down to abundances of $10^{-9}-10^{-8}$ with respect to $\mathrm{H}_{2}$. SO and CS lines have been searched for without success. Line ratios indicate that the molecular emission arises from dense $\left(10^{6}-10^{8} \mathrm{~cm}^{-3}\right)$ and moderately warm $(T \sim 20-40 \mathrm{~K})$ intermediate height regions of the disk atmosphere between the midplane and the upper layer, in accordance with predictions from models of the chemistry in disks. The sizes of the disks were estimated from model fits to the ${ }^{12} \mathrm{CO} 3-2$ line profiles. The abundances of most species are lower than in the envelope around the solarmass protostar IRAS 16293-2422. Freeze-out in the cold midplane and photodissociation by stellar and interstellar ultraviolet photons in the upper layers are likely causes of the depletion. $\mathrm{CN}$ is strongly detected in all disks, and the $\mathrm{CN} / \mathrm{HCN}$ abundance ratio toward the Herbig Ae stars is even higher than that found in galactic photon-dominated regions, testifying to the importance of photodissociation by radiation from the central object in the upper layers. $\mathrm{DCO}^{+}$is detected toward TW Hya, but not in other objects. The high inferred $\mathrm{DCO}^{+} / \mathrm{HCO}^{+}$ratio of $\sim 0.035$ is consistent with models of the deuterium fractionation in disks which include strong depletion of $\mathrm{CO}$. The inferred ionization fraction in the intermediate height regions as deduced from $\mathrm{HCO}^{+}$is at least $10^{-11}-10^{-10}$, comparable to that derived for the midplane from recent $\mathrm{H}_{2} \mathrm{D}^{+}$observations. Comparison with the abundances found in cometary comae is made.
\end{abstract}

Key words. ISM: molecules - stars: circumstellar matter - stars: pre-main-sequence - astrochemistry

\section{Introduction}

The protoplanetary disk phase constitutes a key period in the evolution of matter between the young protostellar and the mature planetary system stages. Before their incorporation into comets and large bodies, the gas and dust could have participated in a complex chemistry within the disk. Studies of the chemistry in disks are therefore important to quantify the chemical composition of protoplanetary material.

The chemical composition of the envelopes around young protostars is now known with increasing detail thanks to the combination of rapid advances in detectors and antenna technology and improved models (e.g., van Dishoeck \& Blake 1998; Langer et al. 2000). Part of this gas and dust settles around the pre-main-sequence star in the form of a disk, and after the collapse and accretion onto the star ceases, planets and comets can form by accumulating gaseous and solid

* Tables 3-5 are only available in electronic form at http://www. edpsciences.org material on timescales of a few million years (e.g., Lissauer 1993; Beckwith \& Sargent 1996; Wuchterl et al. 2000). Surveys from the near-infrared to the millimeter wavelength range have shown that a large fraction of 1-10 million year old Sun-like pre-main-sequence stars harbors a disk in Keplerian rotation (e.g., Beckwith et al. 1990). The masses of these disks $\left(0.001-0.1 M_{\odot}\right)$ is sufficient to form a few giant gaseous planets.

Single-dish and interferometric observations of molecular species other than $\mathrm{CO}$ are starting to reveal the chemistry in disks around classical T Tauri stars (Dutrey et al. 1997; Kastner et al. 1997; Simon et al. 2000; Duvert et al. 2000; van Zadelhoff et al. 2001; Aikawa et al. 2003; Qi et al. 2003; Dartois et al. 2003; Kessler et al. 2003; Wilner et al. 2003). The low- $J$ rotational transitions of simple molecules $\left(\mathrm{HCN}, \mathrm{CN}, \mathrm{HNC}, \mathrm{H}_{2} \mathrm{CO}\right.$, $\left.\mathrm{HCO}^{+}, \mathrm{CS}, \ldots\right)$ are detected, but their abundances relative to $\mathrm{H}_{2}$ are inferred to be orders of magnitude lower than those observed in dark clouds. The prevailing explanation of this depletion involves a combination of freeze-out of the molecules on 
grain surfaces in the cold midplane and their photodissociation by ultraviolet and/or X-rays in the upper atmosphere of disks (see Aikawa et al. 1999a, 2002; Bergin et al. 2003). The abundances are enhanced in the intermediate height regions, which are warm enough for the molecules to remain in the gas phase. Photodesorption induced by ultraviolet radiation (Willacy \& Langer 2000; Westley et al. 1995) or X-rays (Najita et al. 2001) can further populate the upper layers with molecules evaporated from dust grains.

We present here the results of a survey of several lowand high- $J$ molecular transitions observed toward two classical T Tauri stars (LkCa 15 and TW Hya) and two Herbig Ae stars (MWC 480 and HD 163296) using single-dish telescopes. In particular, organic molecules such as $\mathrm{H}_{2} \mathrm{CO}, \mathrm{CH}_{3} \mathrm{OH}$ and $\mathrm{HCN}$ and deuterated species were searched for. The comparison of the two types of objects allows the influence of the color temperature of the radiation field on the chemistry to be studied. There are several advantages in observing high- $J$ transitions over the lower- $J$ ones. First, detections of $\mathrm{CO} J=6 \rightarrow 5$ and $\mathrm{H}_{2}$ show the presence of a warm upper surface layer in protoplanetary disks whose temperature is higher than the freeze-out temperature of most volatile molecules (van Zadelhoff et al. 2001; Thi et al. 2001). Combined with the high densities in disks, this allows the mid- $J$ levels to be readily populated. Models of flaring disks predict that the upper layer facing directly the radiation from the central star can extend out to large radii (Chiang $\&$ Goldreich 1997; D’Alessio et al. 1999). Second, by observing at higher frequencies with single dish telescopes, the lines suffer less beam dilution entailed by the small angular size of disks, typically $1-3^{\prime \prime}$ in radius, than at lower frequencies. Also, confusion with any surrounding low-density cloud material is minimized.

The results for the different molecules are compared to those found for protostellar objects, in particular the solar-mass protostar IRAS 16293-2422, which is considered representative of the initial cloud from which the Sun and the solar nebula were formed. This so-called Class 0 object (André et al. 2000) is younger than the protoplanetary disks studied here, only a few $\times 10^{4} \mathrm{yr}$, and its chemistry is particularly rich as shown by the number of species found in surveys in the (sub)millimeter range (e.g., van Dishoeck et al. 1995; Ceccarelli et al. 2001; Schöier et al. 2002; Cazaux et al. 2003, and references therein). The similarities and differences in the chemical composition between IRAS 16293-2422 and the protoplanetary disks can be used to constrain the chemical models of disks.

At the other extreme, the results for disks can be compared with those found for objects in our solar system, in particular comets. This will provide more insight into the evolution of matter from the protoplanetary disk phase to planetary systems. Unfortunately, the chemical composition of the large bodies in our solar system has changed since their formation 4.6 Gyr ago. For example, solar radiation triggers photochemical reactions in the atmospheres of planets, and the release of energy from the radioactive decay of short-lived elements such as ${ }^{26} \mathrm{Al}$ causes solids to melt. Comets, however, could have kept a record of the chemical composition of the primitive solar nebula because they spent much of their time in the cold outer region of the solar system (the Oort cloud) since their formation (Irvine et al. 2000; Stern 2003). Comparison of cometary $\mathrm{D} / \mathrm{H}$ ratio and the $\mathrm{CH}_{3} \mathrm{OH}$ abundances with those in disks are particularly interesting.

This paper is organized as follows. In Sect. 2, the characteristics of the observed objects are summarized. In Sect. 3, the observational details are provided. The results are given in Sect. 4 where a simple local thermodynamical equilibrium (LTE) and statistical equilibrium analysis is performed. In this section, we also derive several disk characteristics by fitting the ${ }^{12} \mathrm{CO} 3-2$ lines. In Sect. 5, the molecular abundance ratios are discussed. In particular, the $\mathrm{CN} / \mathrm{HCN}$ ratio can trace the photochemistry whereas the $\mathrm{CO} / \mathrm{HCO}^{+}$ratio is a tracer of the fractional ionization. Finally, a discussion on the $\mathrm{D} / \mathrm{H}$ ratio in the disks compared with that found in comets or other star-forming regions is presented (see also van Dishoeck et al. 2003).

\section{Objects}

The sources were selected to have strong $\mathrm{CO} J=3 \rightarrow 2$ fluxes and the highest number of molecular lines detected in previous observations (Qi 2001; Thi et al. 2001; van Zadelhoff et al. 2001). LkCa 15 is a solar mass T Tauri star located in the outer regions of the Taurus cloud. Its age is estimated to be $\sim 10$ million years, although Simon et al. (2000) argue for an age of only 3-5 million years. LkCa 15 is surrounded by a disk whose mass is estimated to be around $0.03 M_{\odot}$, although a higher mass has been obtained from the fitting of its spectral energy distribution (SED) (Chiang et al. 2001). LkCa 15 is one of the strongest millimeter emitting sources in the sample of T Tauri stars surveyed by Beckwith et al. (1990) along with GG Tau and DM Tau.

TW Hya forms part of a young association of stars that has been discovered only recently and is located at only $\sim 56 \mathrm{pc}$ (Webb et al. 1999). TW Hya itself is a classical isolated T Tauri star with a high X-ray flux and a large lithium abundance. Its large $\mathrm{H} \alpha$ equivalent width is indicative of active disk accretion at a rate of $\sim 10^{-8} M_{\odot} \mathrm{yr}^{-1}$ (Kastner et al. 2002). Despite its relatively high age $(\sim 15 \mathrm{Myr})$, TW Hya is surrounded by a disk of mass $\sim 3 \times 10^{-2} M_{\odot}$ (Wilner et al. 2000) seen nearly faceon (Weintraub et al. 1989; Krist et al. 2000; Zuckerman et al. 2000).

MWC 480 and HD 163296 were chosen to be representative of Herbig Ae stars. These two objects have the strongest millimeter continuum emission, with disk masses similar to those around the two T Tauri stars. All selected objects show gas in Keplerian rotation as revealed by CO interferometric observations (Qi 2001; Mannings \& Sargent 1997).

The stellar characteristics of the four objects are given in Table 1 and the disk characteristics in Table 2. In this paper, inclination is defined such that $0^{\circ}$ is a disk viewed pole-on and $90^{\circ}$ edge-on. Detailed modeling of the SED of these objects suggests that the disks are in a state of dust settling, especially LkCa 15 (Chiang et al. 2001). The objects were chosen to be isolated from any cloud material to avoid contamination of the single-dish data. 
Table 1. Stellar characteristics ${ }^{a}$.

\begin{tabular}{lllcllll}
\hline \hline \multicolumn{1}{c}{ Star } & \multicolumn{1}{c}{ SpT } & $\begin{array}{c}\alpha \\
(\mathrm{J} 2000)\end{array}$ & $\begin{array}{c}\delta \\
(\mathrm{J} 2000)\end{array}$ & $\begin{array}{c}D \\
(\mathrm{pc})\end{array}$ & $\begin{array}{c}M_{*} \\
\left(M_{\odot}\right)\end{array}$ & $\log \left(L_{*} / L_{\odot}\right)$ & $\begin{array}{c}\text { Age } \\
(\mathrm{Myr})\end{array}$ \\
\hline LkCa 15 & K7 & 043917.8 & +222103 & 140 & 0.8 & -0.27 & 11.7 \\
TW Hya & K8Ve & 110151.91 & -344217.0 & 56 & 1.0 & -0.60 & $7-15$ \\
HD 163296 & A3Ve & 175621.26 & -215719.5 & 122 & 2.4 & +1.41 & 6.0 \\
MWC 480 & A3ep+sh & 045846.27 & +295037.0 & 131 & 2.2 & +1.51 & 4.6 \\
\hline
\end{tabular}

${ }^{a}$ See Thi et al. (2001) for references. The ages are highly uncertain (see also Simon et al. 2000

Table 2. Disk characteristics.

\begin{tabular}{lccccl}
\hline \hline \multicolumn{1}{c}{ Star } & $\begin{array}{c}\text { Disk mass }^{a} \\
\left(10^{-2} M_{\odot}\right)\end{array}$ & $\begin{array}{c}\text { Radius } \\
(\mathrm{AU})\end{array}$ & $\begin{array}{c}\text { Diameter } \\
\left({ }^{\prime}\right)\end{array}$ & $\begin{array}{c}\text { Inclination } \\
\left({ }^{\circ}\right)\end{array}$ & Ref. \\
\hline LkCa 15 & $3.3 \pm 1.5$ & 425 & 6.2 & $57 \pm 10$ & 1 \\
TW Hya & $3.0 \pm 2.0$ & 200 & 7.0 & $<10$ & $2,3,4,5,6$ \\
HD 163296 & $6.5 \pm 2.9$ & 310 & 5.0 & $32 \pm 5$ & 7 \\
MWC 480 & $2.2 \pm 1.0$ & 695 & 10.4 & $30 \pm 5$ & 7 \\
\hline
\end{tabular}

${ }^{a}$ Total gas + dust mass computed from millimeter continuum flux using a dust opacity coefficient $\kappa_{\lambda}=0.01(1.3 \mathrm{~mm} / \lambda) \mathrm{cm}^{2} \mathrm{~g}^{-1}$ and assuming a standard gas-to-dust mass ratio of 100 .

References. (1) Qi et al. (2003), (2) Weintraub et al. (1989), (3) Krist et al. (2000), (4) Weinberger et al. (2002), (5) Wilner et al. (2000), (6) Calvet et al. (2002), (7) Mannings et al. (1996).

\section{Observations}

The observations were performed between 1998 and 2000 with the James Clerk Maxwell Telescope (JCMT) ${ }^{1}$ located on Mauna Kea for the high- $J$ transitions $(850 \mu \mathrm{m}$ window) and with the 30-m telescope of the Institut de Radioastronomie Millimétrique (IRAM) at Pico Veleta for the lower $J$ lines ( 1 to $3 \mathrm{~mm}$ ). At both telescopes, the observations were acquired in the beam-switching mode with a throw of $120^{\prime \prime}$ at IRAM and $180^{\prime \prime}$ at the JCMT in the azimuth direction. The observations suffer from beam dilution owing to the small projected sizes of the disks of the order of 5-10" in diameter, compared to the beam size of the JCMT (14" at $330 \mathrm{GHz})$ and IRAM (11.3" at $220 \mathrm{GHz}$ ). The data were reduced and analyzed with the SPECX, CLASS and in-house data reduction packages.

The JCMT observations made use of the dual polarization B3 receiver $(315-373 \mathrm{GHz})$ and were obtained mostly in November-December 1999. The antenna temperatures were converted to main-beam temperatures using a beam efficiency of $\eta_{\mathrm{mb}}=0.62$, which was calibrated from observations of planets obtained by the staff at the telescope.

The data were obtained in single sideband mode with the image side band lines reduced in intensity by about $13 \mathrm{~dB}$ (i.e. by a factor of $\sim 20$ ). The sidebands were chosen to minimize the system temperature and to avoid any unwanted emission in the other sideband. The integration times range from $5 \mathrm{~min}$ for the bright ${ }^{12} \mathrm{CO} J=3 \rightarrow 2$ lines to $8 \mathrm{~h}$ for the faint lines to reach a rms noise $\delta T_{\text {ther }}$ of $10-20 \mathrm{mK}$ after binning. The backend was the Digital Autocorrelator Spectrometer (DAS) set at

1 The James Clerk Maxwell Telescope is operated by the Joint Astronomy Centre in Hilo, Hawaii on behalf of the Particle Physics and Astronomy Research Council in the United Kingdom, the National Research Council of Canada and The Netherlands Organization for Scientific Research. a resolution of $\sim 0.15-0.27 \mathrm{~km} \mathrm{~s}^{-1}$ (see Tables 3 and 4), and subsequently Hanning-smoothed to $0.3-0.6 \mathrm{~km} \mathrm{~s}^{-1}$ in spectra where the signal-to-noise ratio is low. Pointing accuracy and focus were regularly checked by observing planets, and was found to be accurate to better than $3^{\prime \prime} \mathrm{rms}$ at the JCMT.

The estimated total rms error $\delta T$ at the JCMT associated with each line is given by the relation (e.g., Papadopoulos \& Seaquist 1998):

$\left(\frac{\delta T}{T_{\mathrm{mb}}}\right)_{\mathrm{tot}}=\left[\left(\frac{\delta T}{T_{\mathrm{mb}}}\right)_{\mathrm{ther}}^{2}+\left(\frac{\delta T}{T_{\mathrm{mb}}}\right)_{\mathrm{drift}}^{2}+\left(\frac{\delta T}{T_{\mathrm{mb}}}\right)_{\mathrm{syst}}^{2}\right]^{1 / 2}$

where the first term on the right-hand side of the relation expresses the ratio between the thermal rms temperature and the main-beam peak temperature averaged over $N_{\text {ch }}$ channels and with a baseline derived from $N_{\text {bas }}$ channels:

$\left(\frac{\delta T}{T_{\mathrm{mb}}}\right)_{\text {ther }}=\frac{\delta T_{\mathrm{ther}}}{T_{\mathrm{A}}^{*}}\left(\frac{N_{\mathrm{bas}}+N_{\mathrm{ch}}}{N_{\mathrm{bas}} N_{\mathrm{ch}}}\right)^{1 / 2}$

where $\delta T_{\text {ther }}$ is the thermal noise per channel and $T_{\mathrm{A}}^{*}$ is the antenna temperature given in Tables 3 and 4 . The beam-switching method gives extremely flat baselines such that $N_{\text {bas }} \gg N_{\text {ch }}$ and

$\left(\frac{\delta T}{T_{\mathrm{mb}}}\right)_{\text {ther }}=\frac{\delta T_{\mathrm{ther}}}{T_{\mathrm{A}}^{*}} \frac{1}{\sqrt{N_{\mathrm{ch}}}} \cdot$

The data are subsequently binned to $N_{\mathrm{ch}}=2$ or 4 , and a line detection is claimed whenever

$\left(\frac{\delta T}{T_{\mathrm{mb}}}\right)_{\text {ther }}<0.3$.

The second therm in Eq. (1) concerns the drift of the temperature scale due to all errors of stochastic nature, and therefore includes any temperature variation of the cold loads or any fluctuation of atmospheric opacity. Measurements of spectral standard sources just before or after the source observations allow 
an estimate of this drift, which is generally found to be $10-15 \%$ and may be up to $20-25 \%$ in difficult parts of the atmospheric window (e.g. $\mathrm{H}_{2} \mathrm{D}^{+}$amd $\mathrm{N}_{2} \mathrm{H}^{+}$lines) depending on the conditions. The lines for which calibration sources are available from measurements by the JCMT staff are mentioned in Table $5^{2}$.

The last term encompasses the systematic error, whose main contributors are the uncertainty in the value of the beamefficiency and pointing errors. As noted above, the pointing at the JCMT was found to be accurate to better than $3^{\prime \prime}$ at $345 \mathrm{GHz}$. Differences in beam efficiencies and pointing should also be reflected in the spectral standard observations, which generally agree within $10-15 \%$ as noted above. This last term is therefore estimated to contribute at most 10-20\%. Taking into account all possible sources of errors, the overall calibration uncertainties can be as high as $30-40 \%$ for a line detected with $3 \sigma$ in a difficult part of the spectrum, whereas it is of order 20-25\% for high $S / N$ lines for which spectral standards have been observed.

As discussed by van Zadelhoff et al. (2001), our $\mathrm{HCO}^{+}$ $J=4-3$ intensity measured in 1999 is a factor of 3 weaker than that obtained by Kastner et al. (1997). More recently, we have re-observed the $\mathrm{HCO}^{+}$line in May 2004 and find intensities on two days which agree with those of Kastner et al. within 10-20\%. For comparison, ${ }^{12}$ CO 3-2 spectra taken in 1999 , 2000 and 2004 are consistent within $10 \%$ with the Kastner et al. results taken in 1995 with a different receiver, as are the HCN 4-3 and CN 3-2 results. Thus, only the $1999 \mathrm{HCO}^{+}$result appears anomalously low, perhaps due to unusually large pointing errors during those observations related to the JCMT "tracking error" problem ${ }^{3}$, unless the ion abundance is variable. We use only the new 2004 data in our analysis. Note that the $\mathrm{H}^{13} \mathrm{CO}^{+}$and $\mathrm{DCO}^{+}$data were taken only 1 week apart so that the analysis of the $\mathrm{DCO}^{+} / \mathrm{HCO}^{+}$ratio should not suffer from any potential long-term variability. Further monitoring of the $\mathrm{HCO}^{+}$line is warranted.

The IRAM-30 $\mathrm{m}$ observations were carried out in December 1998 using the 1-3 mm receivers. The weather conditions were excellent. The three receivers and a splitable correlator were used to observe simultaneously lines at 1.3, 2 and $3 \mathrm{~mm}$. The receivers were tuned single-sideband. Image band rejection was of the order of $10 \mathrm{~dB}$. Forward $\left(F_{\text {eff }}\right)$ efficiencies were measured at the beginning of each run and have been found to be consistent with standard values. We measured $F_{\text {eff }}=0.9,0.82$ and 0.84 at 100,150 and $230 \mathrm{GHz}$ respectively. The derived beam efficiencies $\left(\eta_{\mathrm{mb}}=B_{\mathrm{eff}} / F_{\text {eff }}\right)$ are $0.57,0.69$, and 0.69 at 1,2 and $3 \mathrm{~mm}$ respectively using main-beam efficiencies $\left(B_{\text {eff }}\right)$ provided by the IRAM staff. The pointing and focusing accuracy were regularly checked to ensure pointing errors $<3^{\prime \prime}$ (rms) by observing planets and quasars. TW Hya is unfortunately located too far south to observe with the IRAM $30 \mathrm{~m}$ telescope.

\footnotetext{
${ }^{2}$ See also http://www.jach.hawaii.edu/JACpublic/JCMT/ Heterodyne_observing/Standards/

${ }^{3}$ http://www.jach.hawaii.edu/JCMT/Facility_description/ Pointing/problem_transit.html
}

\section{Results}

\subsection{General characteristics}

The measured antenna temperatures and thermal noise per channel width are summarized in Tables 3 and 4 . The spectra are displayed in Figs. 2 to 4 on the main-beam temperature scale for the four sources. ${ }^{12} \mathrm{CO} J=3 \rightarrow 2$ and ${ }^{13} \mathrm{CO} J=3 \rightarrow 2$ are detected toward all objects. Apart from TW Hya, the profiles of the ${ }^{12} \mathrm{CO} J=3 \rightarrow 2$ spectra are double-peaked with peak separations of $\sim 2 \mathrm{~km} \mathrm{~s}^{-1}$ for both three objects. The ${ }^{12} \mathrm{CO}$ $J=3 \rightarrow 2$ spectrum of MWC 480 shows a profile with slightly different peak strengths. However, the level of asymmetry is not signifcant compared to the noise. ${ }^{12} \mathrm{CO} J=3 \rightarrow 2$ observations obtained with $30^{\prime \prime}$ offsets and position-switching to an emission-free position are shown in Fig. 5 for the four objects. The maps around LkCa 15, TW Hya and MWC 480 confirm that these objects are isolated from cloud material. The observations at offset positions from HD 163296 show emission at velocities shifted compared with the velocity of the star. The extinction to HD 163296 is sufficiently low that the extended low density emission is unlikely to arise from a foreground cloud. The offset emission is only seen in ${ }^{12} \mathrm{CO}$, not in ${ }^{13} \mathrm{CO}$ or other molecules. Lines arising from high- $J$ transitions require high critical densities and are therefore not likely to come from a low density cloud. A more complete discussion on the possible contamination by foreground and/or background clouds is given in Thi et al. (2001).

High- $J$ lines of various molecules are detected in the disks. Lines with high signal-to-noise ratio toward $\mathrm{LkCa} 15$, HD 163296 and MWC 480 show a double-peak structure corresponding to emission from a disk in Keplerian rotation viewed under an inclination angle $i$ (Beckwith \& Sargent 1993). The line profiles observed toward TW Hya are well fitted by a single gaussian, consistent with a disk seen almost face-on. The profiles show no evidence of extended velocity wings characteristic of molecular outflows in any of the objects. The velocity integrated main-beam temperatures for the four sources are summarized in Table 6. This table includes the energy of the upper level of the transitions, their critical densities and frequencies, the telescope at which they were observed and the beam size. The critical densities are defined as $n_{\mathrm{cr}}=A_{u l} / \sum_{l} q_{u l}$, where $A_{u l}$ is the Einstein $A$ coefficient of the transition $u \rightarrow l$ and $q_{u l}$ the downward rate coefficient. They have been computed in the optically thin limit at $100 \mathrm{~K}$ using the molecular data listed in Jansen et al. (1994) and Jansen (1995). For optically thick lines, the critical densities are lowered by roughly the optical depth of the line.

The upper limits are computed assuming a main beam temperature which corresponds to twice the rms noise level in a $0.3 \mathrm{~km} \mathrm{~s}^{-1}$ bin and a line profile similar to that derived from fitting the ${ }^{13} \mathrm{CO} J=3 \rightarrow 2$ lines.

The ion $\mathrm{HCO}^{+}$is detected in all sources. Toward TW Hya, $\mathrm{H}^{13} \mathrm{CO}^{+}$is also seen, and the ratio of integrated fluxes $\mathrm{HCO}^{+} / \mathrm{H}^{13} \mathrm{CO}^{+}$of 24 is lower than the interstellar isotopic ratio $\left[{ }^{12} \mathrm{C}\right] /\left[{ }^{13} \mathrm{C}\right] \sim 60$, indicating that $\mathrm{HCO}^{+}$line is optically thick (van Zadelhoff et al. 2001). Emission from the $\mathrm{CN}$ radical in the $J=3 \rightarrow 2$ line is surprisingly strong in all objects 

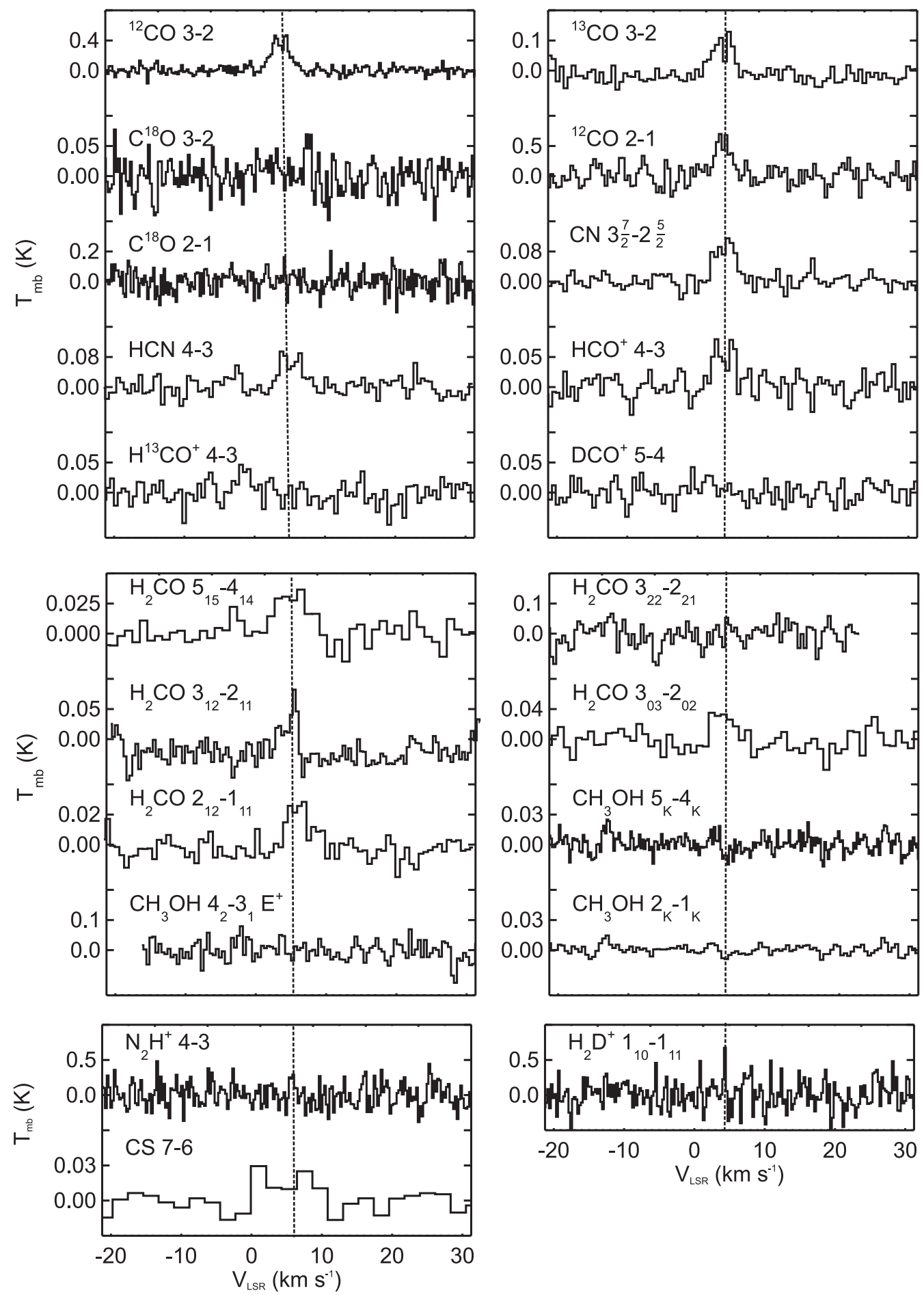

Fig. 1. Line profiles observed toward LkCa15. The dashed line indicates the velocity of the source. Note the different antenna temperature scales for the different features.

compared to $\mathrm{HCN} J=4 \rightarrow 3$. HNC $J=4 \rightarrow 3$ was only searched toward TW Hya and has not been detected. The line intensities for some species in this object differ with previous observations by Kastner et al. (1997) (see van Zadelhoff et al. 2001, Sect. 3). In general, the two Herbig Ae stars display a less rich chemistry than the two classical $\mathrm{T}$ Tauri stars. In particular, $\mathrm{HCN}$ is not detected in either source in our observations. Qi (2001), however, reports detection of HCN $J=1 \rightarrow 0$ toward MWC 480 with the Owens Valley Millimeter Array (OVRO).
Several lines of $\mathrm{H}_{2} \mathrm{CO}$ are seen toward $\mathrm{LkCa} 15$ with the IRAM 30-m and JCMT, but not toward the other three disks. Deep searches for various $\mathrm{CH}_{3} \mathrm{OH}$ lines with the IRAM 30-m and JCMT down to very low noise levels did not yield any detections. So far, $\mathrm{CH}_{3} \mathrm{OH}$ has only been seen toward $\mathrm{LkCa} 15$ through its $J=5_{K} \rightarrow 4_{K}$ and $J=4_{23} \rightarrow 3_{13}$ lines using OVRO (Qi 2001). This illustrates the power of interferometers to detect minor species in disks, because, with a beam diameter of $1^{\prime \prime}-2^{\prime \prime}$, the fluxes are much less beam-diluted, in addition to a 

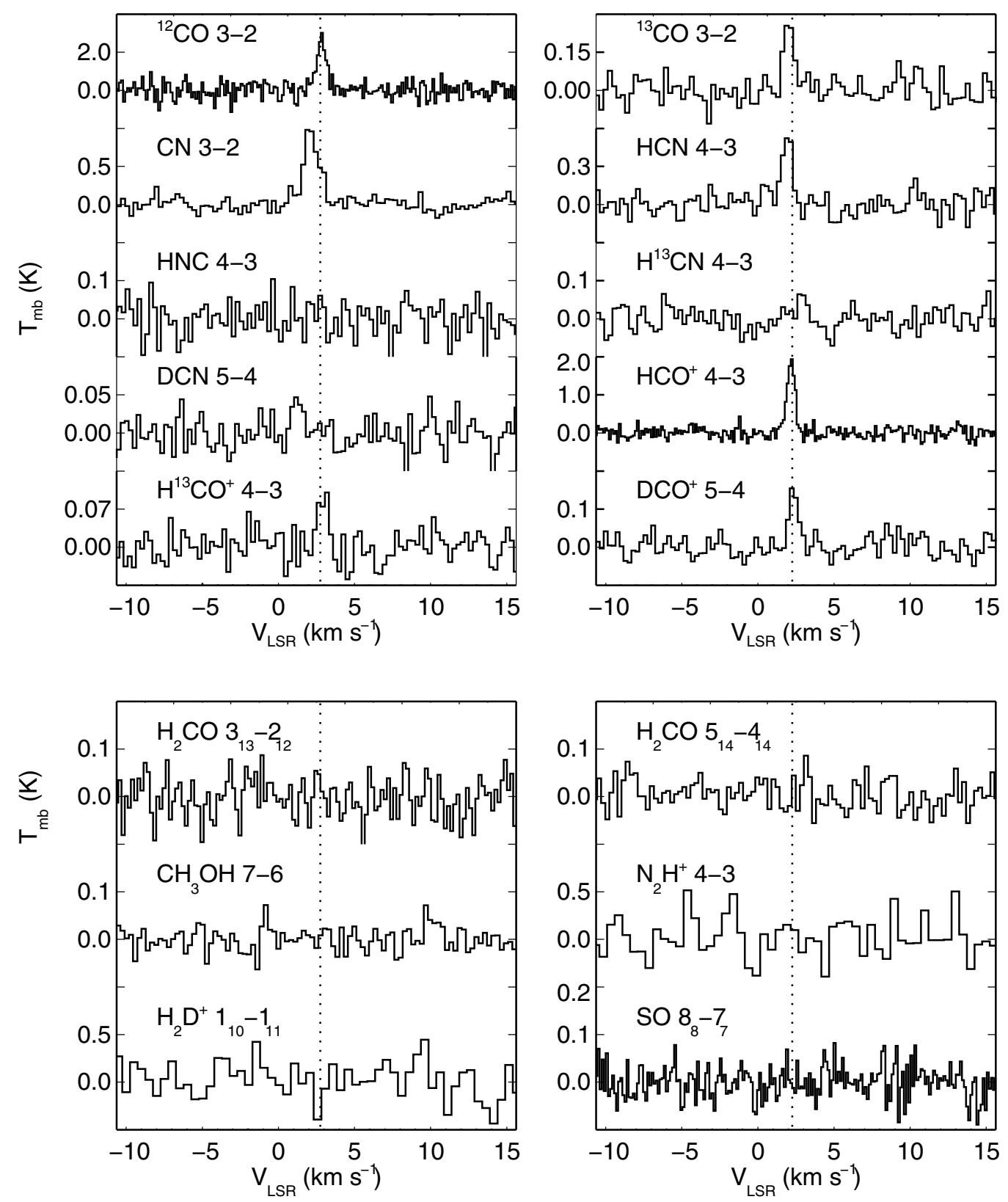

Fig. 2. Line profiles observed toward TW Hya. Note the different antenna temperature scales for the different features.

potentially larger collecting area when a great number of dishes is available.

No CS $J=7 \rightarrow 6$ line nor lines of $\mathrm{SO}_{2}$, some of which occur fortuitously in other settings (e.g., near $\mathrm{H}_{2} \mathrm{CO} 351 \mathrm{GHz}$ ), were detected toward LkCa 15. A deep limit on SO was obtained toward TW Hya (Fig. 2 and Table 6).

Finally, $\mathrm{DCO}^{+}$is detected for the first time in a disk, as reported by van Dishoeck et al. (2003). The $\mathrm{DCO}^{+} J=5 \rightarrow 4$ line is observed toward TW Hya with a strength similar to that of $\mathrm{H}^{13} \mathrm{CO}^{+} J=4 \rightarrow 3$, but the line is not detected toward LkCa 15 and MWC 480, where $\mathrm{H}^{13} \mathrm{CO}^{+}$is also not seen. There is a hint of a feature near the DCN $J=5 \rightarrow 4$ line toward TW Hya, but it is offset by a few $\mathrm{km} \mathrm{s}^{-1}$. Deeper integrations or interferometer data are needed to confirm this. Note also the high critical density needed to excite the DCN $J=5 \rightarrow 4$ line $\left(\sim 4.8 \times 10^{7} \mathrm{~cm}^{-3}\right)$, which may make it more difficult to detect than $\mathrm{DCO}^{+}$. The ground-state line of ortho$\mathrm{H}_{2} \mathrm{D}^{+}$at $372 \mathrm{GHz}$ was searched toward three sources (LkCa 15, TW Hya and MWC 480) in a setting together with $\mathrm{N}_{2} \mathrm{H}^{+}$, but neither was detected. Because of the poor atmosphere and higher receiver noise at this frequency, the limits for both $\mathrm{H}_{2} \mathrm{D}^{+}$and $\mathrm{N}_{2} \mathrm{H}^{+}$are not very deep, except toward MWC 480 . Recently, Ceccarelli et al. (2004) have published the detection of the $\mathrm{H}_{2} \mathrm{D}^{+} 372 \mathrm{GHz}$ line from the DM Tau disk using the Caltech Submillimeter Observatory, together with a tentative feature from the TW Hya disk. Their integrated line intensity toward TW Hya is $\int T_{\mathrm{MB}} \mathrm{d} V=0.39 \pm 0.12 \mathrm{~K} \mathrm{~km} \mathrm{~s}^{-1}$, compared with our $2 \sigma$ limit of $0.20 \mathrm{~K} \mathrm{~km} \mathrm{~s}^{-1}$. Taking into account the smaller beam dilution in the JCMT beam and the measurement uncertainties, the difference between these two data sets is about a factor of two. 

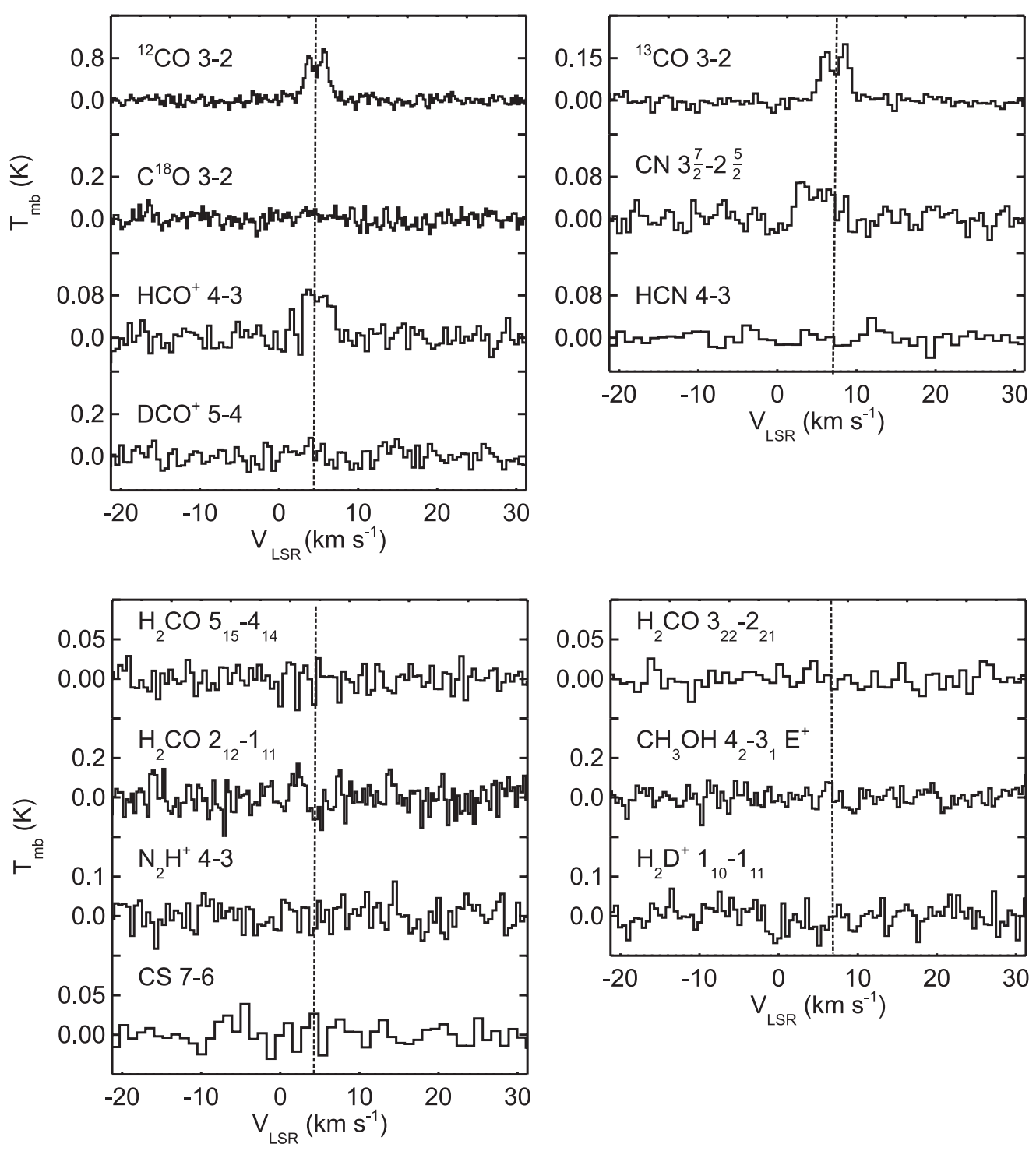

Fig. 3. Line profiles observed toward MWC 480. Note the different antenna temperature scales for the different features.

\subsection{Disks properties and molecular abundances}

\subsubsection{Disk mean density}

The mean density can be constrained from line ratios of molecules with high dipole moments such as $\mathrm{HCO}^{+}, \mathrm{CN}, \mathrm{HCN}$ or $\mathrm{H}_{2} \mathrm{CO}$. A simple excitation analysis was performed using an escape probability code described in Jansen et al. $(1994,1995)$. The code computes the statistical equilibrium population of the rotational levels given the kinetic temperature, volumn density and column density. Integrated temperatures of low- $J$ transitions from Qi (2001) were used to complement our high- $J$ data. Both sets of data were corrected for beam dilution by multiplying the observed velocity integrated main-beam temperatures by the size of the beam as listed in Table 6 . The analysis for LkCa 15 and TW Hya has been performed previously by van Zadelhoff et al. (2001) using $\mathrm{HCO}^{+}$and $\mathrm{HCN}$, and takes both the radial and vertical density structure of the disk into account. Here $\mathrm{H}_{2} \mathrm{CO}$ is also used as a diagnostic for LkCa 15 adopting the same method. Consistent with their results, we find that the densities in the regions probed by our observations range from $10^{6}$ to $10^{8} \mathrm{~cm}^{-3}$. This density refers to the region where the molecular lines are emitted. The fractions of mass in a given density interval for various disk models are shown in Fig. 3 of van Zadelhoff et al. (2001). In all models (Chiang \& Goldreich 1997; D'Alessio et al. 1999; Bell et al. 1997), most of the gas is located in the region of the disk where the density is greater than $10^{6} \mathrm{~cm}^{-3}$. Those densities are sufficient for most transitions studied here to be thermalized. We refer to the paper of van Zadelhoff et al. (2001) for a detailed discussion on the disk models, the densities derived from line ratios and the disk location where the lines are expected to become optically thick.

\subsubsection{Disk mean temperature}

The mean kinetic temperatures are less well constrained: the ratio ${ }^{13} \mathrm{CO} J=3 \rightarrow 2 /{ }^{13} \mathrm{CO} J=1 \rightarrow 0$ of $1.35 \pm 0.4$ suggests that $T_{\text {kin }} \sim 20-40 \mathrm{~K}$ for $\mathrm{LkCa} 15$ in the region where the ${ }^{13} \mathrm{CO}$ emission originates, assuming that both lines are optically thin (van Zadelhoff et al. 2001). The bulk material where CO emits 

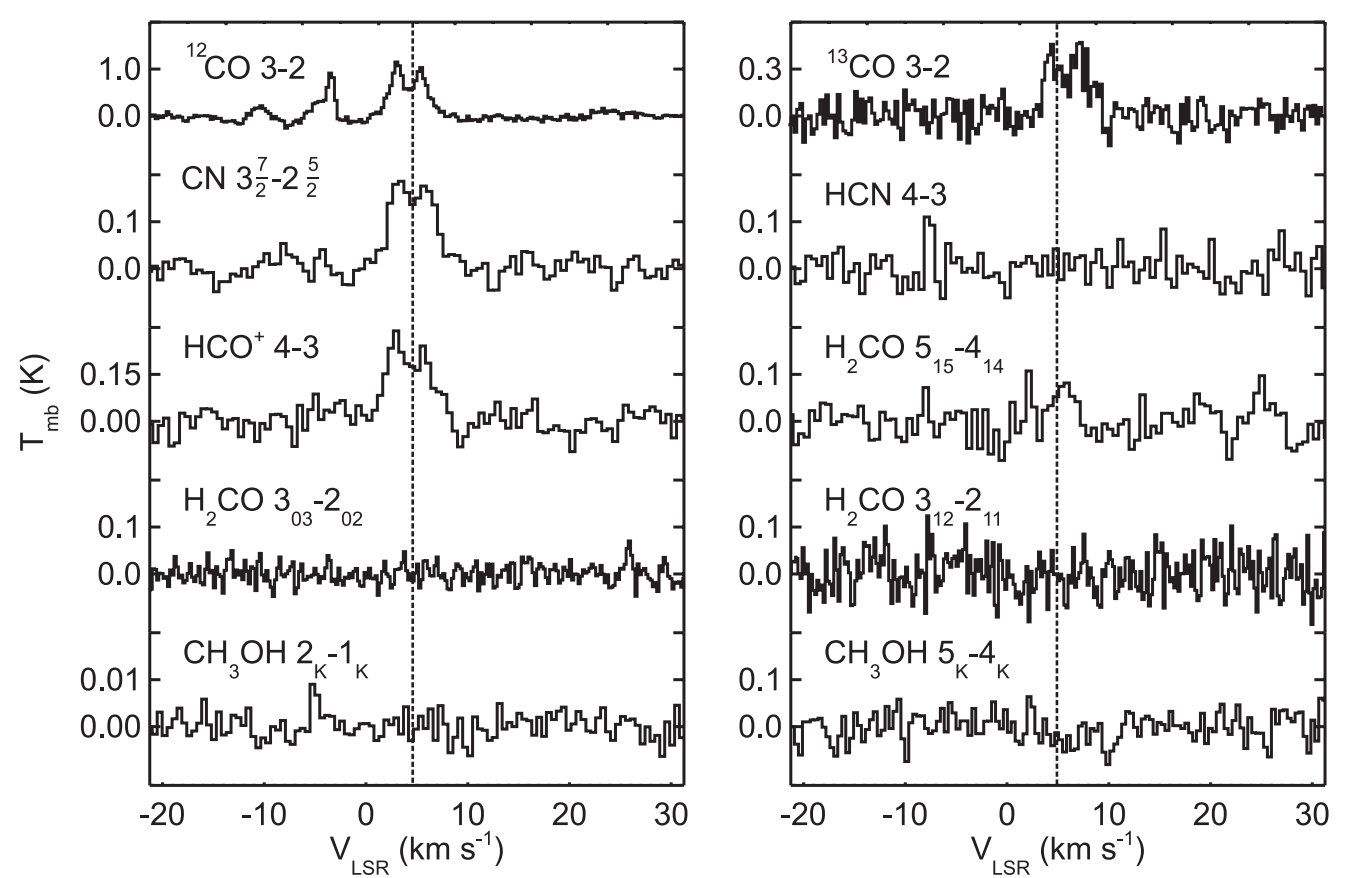

Fig. 4. Line profiles observed toward HD 163296. Note the different antenna temperature scales for the different features.
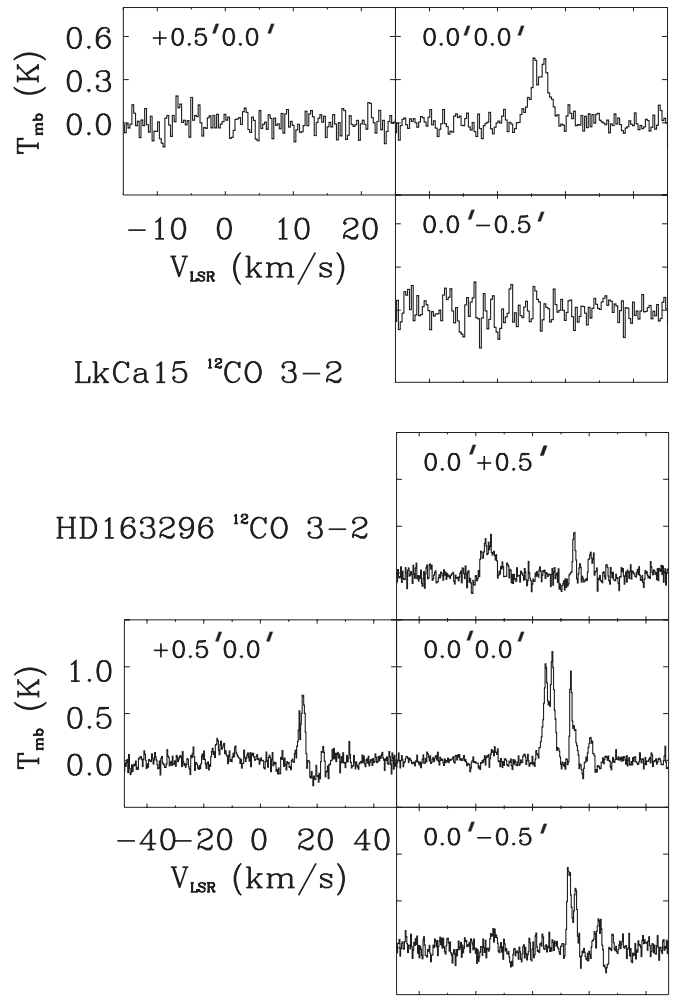
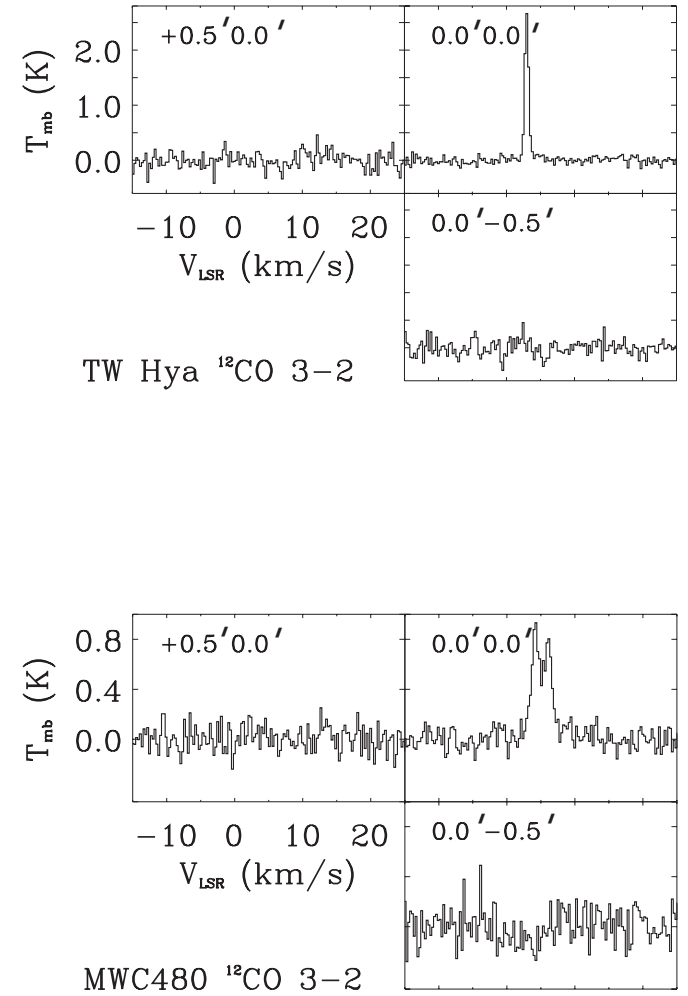

Fig. 5. ${ }^{12} \mathrm{CO} J=3 \rightarrow 2$ maps toward TW Hya, LkCa 15, HD 163296 and MWC 480.

is therefore on average moderately warm and the density is high enough that the level populations can be assumed to be thermalized for most cases. The ratios of $2.4 \pm 0.7$ for MWC 480 and $1.7 \pm 0.5$ for HD 163296 indicate similar temperature ranges. From the $\mathrm{CO} J=6 \rightarrow 5 / J=3 \rightarrow 2$ ratios presented in Thi et al. (2001), it is found that the upper layers of disks have temperatures in the range $25-60 \mathrm{~K}$. The gas temperatures derived from the $\mathrm{H}_{2}$ data in Thi et al. (2001) are slightly higher for disks around Herbig Ae stars than around T Tauri stars, as expected if the disks are heated by the radiation from the central star. The $\mathrm{H}_{2} \mathrm{CO} J=3_{03} \rightarrow 2_{02} / J=3_{22} \rightarrow 2_{21}$ ratio is potentially a good temperature indicator, but the $J=3_{03} \rightarrow 2_{02}$ line has only been detected toward $\mathrm{LkCa} 15$. The limit on the line ratio 
Table 6. Integrated line intensities.

\begin{tabular}{|c|c|c|c|c|c|c|c|c|c|c|}
\hline \multirow[b]{2}{*}{ Line } & \multirow[b]{2}{*}{$\begin{array}{c}E_{\text {upper }} \\
(\mathrm{K})\end{array}$} & \multirow[b]{2}{*}{$\begin{array}{c}n_{\text {crit }}^{a} \\
\left(\mathrm{~cm}^{-3}\right)\end{array}$} & \multirow[b]{2}{*}{$\begin{array}{c}v \\
(\mathrm{GHz})\end{array}$} & \multirow[b]{2}{*}{ Telescope } & \multirow[b]{2}{*}{$\begin{array}{c}\text { Beam } \\
\left({ }^{\prime \prime}\right)\end{array}$} & \multirow[b]{2}{*}{$\mathrm{Cal}^{d}{ }^{d}$} & \multicolumn{4}{|c|}{$\int T_{\mathrm{mb}} \mathrm{d} v\left(\mathrm{~K} \mathrm{~km} \mathrm{~s}^{-1}\right)$} \\
\hline & & & & & & & LkCa15 & TW Hya & HD 163296 & MWC 480 \\
\hline${ }^{12} \mathrm{CO} J=2 \rightarrow 1$ & 16.6 & $2.7(3)$ & 230.538 & IRAM30 m & 10.7 & $\ldots$ & 1.82 & $\ldots$ & $\ldots$ & $\ldots$ \\
\hline${ }^{12} \mathrm{CO} J=3 \rightarrow 2$ & 33.2 & $8.4(3)$ & 345.796 & JCMT & 13.7 & yes & 1.17 & 1.98 & 3.78 & 2.88 \\
\hline${ }^{13} \mathrm{CO} J=3 \rightarrow 2$ & 31.7 & $8.4(3)$ & 330.587 & JCMT & 14.3 & yes & 0.39 & 0.24 & 0.94 & 0.57 \\
\hline $\mathrm{C}^{18} \mathrm{O} J=2 \rightarrow 1$ & 15.8 & $2.7(3)$ & 219.560 & JCMT & 21.5 & yes & $<0.20$ & $\ldots$ & $\ldots$ & $\ldots$ \\
\hline $\mathrm{C}^{18} \mathrm{O} J=3 \rightarrow 2$ & 31.6 & $8.4(3)$ & 329.330 & JCMT & 14.3 & yes & $<0.14$ & $\ldots$ & $\ldots$ & $\ldots$ \\
\hline $\mathrm{HCO}^{+} J=4 \rightarrow 3$ & 42.8 & $1.8(6)$ & 356.734 & JCMT & 13.2 & yes & 0.26 & 1.26 & 1.10 & 0.35 \\
\hline $\mathrm{H}^{13} \mathrm{CO}^{+} J=4 \rightarrow 3$ & 41.6 & $1.8(6)$ & 346.998 & JCMT & 13.6 & yes & $<0.13$ & 0.07 & $\ldots$ & $\ldots$ \\
\hline $\mathrm{DCO}^{+} J=5 \rightarrow 4$ & 51.8 & $3.0(6)$ & 360.169 & JCMT & 13.1 & yes & $<0.10$ & 0.11 & $\ldots$ & $\ldots$ \\
\hline $\mathrm{CN} J=3 \frac{7}{2} \rightarrow 2 \frac{5}{2}$ & 32.7 & $6.0(6)$ & 340.248 & JCMT & 13.9 & no & 0.67 & 1.14 & 0.95 & 0.29 \\
\hline $\mathrm{HCN} J=4 \rightarrow 3$ & 42.5 & $8.5(6)$ & 354.506 & JCMT & 13.3 & yes & 0.25 & 0.49 & $<0.20$ & $<0.07$ \\
\hline $\mathrm{H}^{13} \mathrm{CN} J=4 \rightarrow 3$ & 41.4 & $8.5(6)$ & 345.339 & JCMT & 13.6 & no & $\ldots$ & $<0.04$ & $\ldots$ & $\ldots$ \\
\hline HNC $J=4 \rightarrow 3$ & 43.5 & $8.5(6)$ & 362.630 & JCMT & 13.0 & no & $\ldots$ & $<0.05$ & $\ldots$ & $\ldots$ \\
\hline $\mathrm{DCN} J=5 \rightarrow 4$ & 52.1 & $4.8(7)^{b}$ & 362.046 & JCMT & 13.0 & yes & $\ldots$ & $<0.03$ & $\ldots$ & $\ldots$ \\
\hline $\mathrm{CS} J=7 \rightarrow 6$ & 65.8 & $2.9(6)$ & 342.883 & JCMT & 14.0 & no & $<0.08$ & $\ldots$ & $\ldots$ & $<0.08$ \\
\hline SO $J=8_{8} \rightarrow 7_{7}$ & 87.7 & $1.8(6)$ & 344.310 & JCMT & 13.7 & no & $\cdots$ & $<0.10$ & $\cdots$ & $\cdots$ \\
\hline $\mathrm{H}_{2} \mathrm{CO} J=2_{12} \rightarrow 1_{11}$ & 21.9 & $1.0(5)$ & 140.839 & IRAM30 m & 17.5 & $\ldots$ & 0.17 & $\ldots$ & $<0.10$ & $<0.40$ \\
\hline $\mathrm{H}_{2} \mathrm{CO} \mathrm{J}=3_{03} \rightarrow 2_{02}$ & 21.0 & $4.7(5)$ & 218.222 & IRAM30 m & 11.3 & $\ldots$ & 0.14 & $\ldots$ & $<0.30$ & $\ldots$ \\
\hline $\mathrm{H}_{2} \mathrm{CO} J=3_{22} \rightarrow 2_{21}$ & 68.1 & $2.3(5)$ & 218.475 & IRAM30 m & 11.3 & $\ldots$ & $<0.10$ & $\ldots$ & $\ldots$ & $<0.06$ \\
\hline $\mathrm{H}_{2} \mathrm{CO} J=3_{12} \rightarrow 2_{11}$ & 33.5 & $4.5(5)$ & 225.697 & IRAM30 m & 10.9 & $\ldots$ & 0.10 & $\ldots$ & $<0.30$ & $\ldots$ \\
\hline $\mathrm{H}_{2} \mathrm{CO} J=3_{12} \rightarrow 2_{11}$ & 33.5 & $4.5(5)$ & 225.697 & JCMT & 22.2 & no & $\ldots$ & $<0.05$ & $\ldots$ & $\ldots$ \\
\hline $\mathrm{H}_{2} \mathrm{CO} \mathrm{J}=5_{15} \rightarrow 4_{14}$ & 62.5 & $1.7(6)$ & 351.768 & JCMT & 13.4 & yes & 0.29 & $<0.04$ & $<0.20$ & $<0.09$ \\
\hline $\mathrm{CH}_{3} \mathrm{OH} J=2_{K} \rightarrow 1_{K}$ & 6.9 & $2.6(3)^{c}$ & 96.741 & IRAM-30 m & 25.4 & $\ldots$ & $<0.05$ & $\ldots$ & $<0.03$ & $\ldots$ \\
\hline $\mathrm{CH}_{3} \mathrm{OH} J=4_{2} \rightarrow 3_{1} \mathrm{E}^{+}$ & 45.4 & $3.7(4)$ & 218.440 & IRAM30 m & 11.3 & $\ldots$ & $<0.10$ & $\ldots$ & $\ldots$ & $<0.20$ \\
\hline $\mathrm{CH}_{3} \mathrm{OH} J=5_{K} \rightarrow 4_{K}$ & 34.8 & $4.5(4)$ & 241.791 & IRAM30 m & 10.2 & $\ldots$ & $<0.10$ & $\ldots$ & $<0.10$ & $\ldots$ \\
\hline $\mathrm{CH}_{3} \mathrm{OH} J=7_{K} \rightarrow 6_{K}$ & 65.0 & $1.3(5)$ & 338.409 & JCMT & 13.9 & yes & $\ldots$ & $<0.02$ & $\ldots$ & $\ldots$ \\
\hline $\mathrm{N}_{2} \mathrm{H}^{+} J=4 \rightarrow 3$ & 44.7 & $4.4(6)$ & 372.672 & JCMT & 12.7 & no & $<0.10$ & $<0.30$ & $\ldots$ & $<0.05$ \\
\hline $\mathrm{H}_{2} \mathrm{D}^{+} J=1_{10} \rightarrow 1_{11}$ & 104.3 & $1.2(6)$ & 372.421 & JCMT & 12.7 & no & $<0.10$ & $<0.20$ & $\ldots$ & $<0.05$ \\
\hline
\end{tabular}

Note. The dots indicate not observed. When a line is not detected, a $2 \sigma$ upper limit on $T_{\mathrm{mb}}$ in a $0.3 \mathrm{~km} \mathrm{~s}^{-1}$ bin is computed and the same profile as the ${ }^{13} \mathrm{CO} J=3 \rightarrow 2$ line is assumed. The beam size $(H P B W)$ is computed for the IRAM-30 $\mathrm{m}$ using the fitting formula $H P B W\left({ }^{\prime \prime}\right)=$ 2460/frequency $(\mathrm{GHz}) .{ }^{a}$ Unless specified, the critical densities are taken from Jansen (1995) for $T_{\text {kin }}=100 \mathrm{~K}$ assuming optically thin lines. $a(b)$ means a $\times 10^{b} .{ }^{b}$ Computed using the collisional rate coefficients for HCN. ${ }^{c}$ Derived assuming the collisional rate coefficients of Peng \& Whiteoak (1993). ${ }^{d}$ Observation of calibration sources before and/or after the object. More details are given in Table 5.

constrains the temperature to be below $200 \mathrm{~K}$. A mean temperature of $25 \mathrm{~K}$ is adopted in the remaining parts of the paper.

\subsubsection{Disk size}

The disk sizes are important ingredients for comparing the observed column densities with models. Since sizes are notoriously difficult to derive from low $\mathrm{S} / \mathrm{N}$ interferometer maps, an attempt has been made to infer them directly from our model profiles. Two methods have been employed. First, since the ${ }^{12} \mathrm{CO} 3-2$ emission line is optically thick, it probes the surface temperature profile of the disk (van Zadelhoff et al. 2001).
Using the method described by Dutrey et al. (1997) an estimate of the disk size can be made from the ${ }^{12} \mathrm{CO} J=3 \rightarrow 2$ lines:

$$
\int T_{\mathrm{mb}} \mathrm{d} v=T_{\mathrm{ex}}(\rho \delta v)\left[\frac{\pi\left(R_{\mathrm{out}}^{2}-R_{\mathrm{in}}^{2}\right)}{D^{2}} \cos i\right] \Omega_{\mathrm{a}}^{-1}
$$

where $R_{\text {in }}$ and $R_{\text {out }}$ are the inner and outer radii, $\delta v$ is the local turbulent velocity (between 0.1 and $0.2 \mathrm{~km} \mathrm{~s}^{-1}$ ) and $\rho$ a geometrical factor of the order of 1.5. We adopt here $\rho \delta v=$ $0.3 \mathrm{~km} \mathrm{~s}^{-1}, R_{\text {in }}=0 \mathrm{AU}, T_{\text {ex }}=25,30$, and $50 \mathrm{~K}$ as the mean disk excitation temperature. The values for the inclinations $i$, distances $D$ (in AU) and beam sizes $\Omega_{\mathrm{a}}$ are provided in Tables 1 and 2. The derived disk sizes are given in Table 7 . 
Our estimates for $T_{\mathrm{ex}}=30 \mathrm{~K}$ are similar to published values except for HD 163296, which we find to have twice the size found by Mannings \& Sargent (1997), who measured it directly from their ${ }^{12} \mathrm{CO} 1-0$ map. Spectra of ${ }^{12} \mathrm{CO} 3-2$ emission line were also generated using a standard parametric disk model as described by, e.g., Beckwith \& Sargent (1993). The code uses a ray-tracing method and assumes that the population of the rotational levels is in Local Thermodynamic Equilibrium. All disks have a power-law density profile of the form $n(r)=n_{0}(r / 1 \mathrm{AU})^{-2.5}$. The exact value of $n_{0}$ cannot be constrained by fitting optically thick lines and we assume a typical value of $5 \times 10^{13} \mathrm{~cm}^{-3}$ at $1 \mathrm{AU}$ in the mid-plane. The disk is in hydrostatic equilibrium in the vertical direction. The inner radius is set to $0.01 \mathrm{AU}$ and is not a significant parameter. The free parameters of this model are the excitation temperature $T_{\text {in }}$ at $1 \mathrm{AU}$, the inclination $i$ and the outer radius $R_{\text {out }}$. For simplicity, the gas temperature in the disk is assumed to have a radial profile power index of 0.5 and isothermal in the vertical direction. The best fits are found using a downhill simplex method (e.g., Press et al. 1997). Figure 6 shows the observed spectra and their best fits obtained with the parameters reported in Table 7 . The outer radii found by this ray-tracing model are smaller than those from the optical depth model with $T_{\text {ex }}=30 \mathrm{~K}$, which can be ascribed to additional contributions from warmer gas at large radii not taken account in the isothermal disk model. Note that $T_{\text {in }}$ and $R_{\text {out }}$ are probably degenerate: Table 7 gives two sets of values for $\mathrm{LkCa} 15$ that can both fit the spectra. Only high signal-to-noise spatially resolved interferometer images can lift this degeneracy. The larger outer radius (and smaller inner radius temperature) is adopted, which is closer to that found by direct fitting of interferometric maps (Qi et al. 2003). The inclinations are consistent with published values (see Table 2).

\subsection{Column densities}

Given the sizes, molecular column densities can be derived from the observed line strengths. Two additional assumptions need to be made: the excitation temperature and the line optical depth. The line ratio analysis shows that the lines arise from sufficiently high density regions $\left(10^{5}-10^{7} \mathrm{~cm}^{-3}\right)$ that they can be assumed to be thermalized to first order, although small deviations are expected in the surface layers (see below). Therefore, a single excitation temperature of $25 \mathrm{~K}$ is adopted to allow easy comparison between the disks.

The optical depth can be estimated from the ratio of lines from two isotopologues, assuming that the two species have the same excitation temperature. Such data are available for a few species and lines, and the results are summarized in Table 8. It is seen that both the ${ }^{12} \mathrm{CO}$ and $\mathrm{H}^{12} \mathrm{CO}^{+}$lines are very optically thick. An alternative method is to compare the size of the optically thick blackbody which accounts for the line flux to the actual disk radius derived from the optically thick ${ }^{12} \mathrm{CO}$ line. We adopt again the approach of Dutrey et al. (1997) and rewrite Eq. (5) as follows:

$$
R_{\text {line }}(\mathrm{AU})=106.4\left(\frac{\theta_{\mathrm{a}}}{1^{\prime \prime}}\right)\left(\frac{D}{100 \mathrm{pc}}\right) \sqrt{\frac{\int T_{\mathrm{mb}} \mathrm{d} v}{T_{\mathrm{ex}} \rho \delta v} \times \frac{1}{\pi \cos i}}
$$

where $\theta_{\mathrm{a}}$ is the main-beam diameter at half power in arcsec. For a Gaussian shape, the solid angle is given by $\Omega_{\mathrm{a}}=1.133 \theta_{\mathrm{a}}^{2}$.

Assuming $T_{\mathrm{ex}}=25 \mathrm{~K}$, all derived radii are significantly smaller than the $\mathrm{CO}$ disk sizes, except for $\mathrm{CN} J=3 \frac{7}{2} \rightarrow 2 \frac{5}{2}$. This would suggest that the lines from species other than CO are less thick in the outer parts of the disks $(R>300 \mathrm{AU})$ to which our data are most sensitive, but we consider the direct determination through the isotopologue ratios more reliable.

When the medium is slightly optically thick $(\tau<3)$ and $T_{\text {ex }} \gg T_{\mathrm{CMB}}$ with $T_{\mathrm{CMB}}=2.73 \mathrm{~K}$, the column density of the upper level $N_{\mathrm{u}}$ is given by:

$N_{\mathrm{u}}=\frac{8 \pi k v^{2} W}{h c^{3} A_{u l}}\left(\frac{\Omega_{\mathrm{a}}+\Omega_{\mathrm{s}}}{\Omega_{\mathrm{s}}}\right)\left\langle\frac{\tau}{1-\mathrm{e}^{-\tau}}\right\rangle$

where $v$ is the frequency of the transition, $\tau$ is the mean optical depth, $W=\int T_{\mathrm{mb}} \mathrm{d} v$ is the integrated line intensity expressed in $\mathrm{K} \mathrm{km} \mathrm{s}^{-1}$, and $\Omega_{\mathrm{a}}$ and $\Omega_{\mathrm{s}}$ are the telescope main-beam and the source solid angles, respectively. The ratio $\left(\Omega_{\mathrm{a}}+\Omega_{\mathrm{s}}\right) / \Omega_{\mathrm{s}}$ is the beam dilution factor. Since the inferred disk sizes are much smaller than the beam sizes, $\left(\Omega_{\mathrm{a}}+\Omega_{\mathrm{s}}\right) / \Omega_{\mathrm{s}} \simeq \Omega_{\mathrm{a}} / \Omega_{\mathrm{s}}$. The Einstein $A_{\mathrm{ul}}$ coefficient of the transition in units of $\mathrm{s}^{-1}$ coefficient is given by:

$A_{u l}=\left(\frac{64 \pi^{4} v^{3}}{3 h c^{3}}\right) \frac{S \mu^{2}}{g_{u}}$

where $\mu$ is the dipole moment of the molecule in Debye, $S$ is the line strength, and $g_{\mathrm{u}}=g_{J} g_{K} g_{I}$ is the statistical weight of the upper level. Finally, the factor

$\beta^{-1}=\left\langle\frac{\tau}{1-\mathrm{e}^{-\tau}}\right\rangle$

is the escape probability in the so-called Sobolev or Large Velocity Gradient approximation.

The column density in level $u$ is related to the total column density $N$ by:

$N_{\mathrm{u}}=\frac{N}{Q_{\mathrm{rot}}\left(T_{\mathrm{ex}}\right)} g_{J} g_{K} g_{I} \mathrm{e}^{-E_{\mathrm{u}} / T_{\mathrm{ex}}}$

where $Q_{\text {rot }}\left(T_{\text {ex }}\right)$ is the rotational partition function, $g_{J}$ is the rotational statistical weight factor equal to $2 J+1$ for diatomic or linear molecules, $g_{K}$ is the $K$-level degeneracy, $g_{\mathrm{I}}$ is the reduced nuclear spin degeneracy, and $E_{\mathrm{u}}$ is the energy of the upper level expressed in K. For linear molecules, $g_{K}=g_{I}=1$ for all levels. Formaldehyde, $\mathrm{H}_{2} \mathrm{CO}$, is an asymmetric top molecule with $I=$ $1 / 2$. Combining the above equations, the total column density can be expressed as:

$$
\begin{aligned}
N= & \frac{1.67 \times 10^{14}}{v \mu^{2} S} Q_{\mathrm{rot}}\left(T_{\mathrm{ex}}\right) \mathrm{e}^{E_{\mathrm{u}} / T_{\mathrm{ex}}}\left\langle\frac{\tau}{1-\mathrm{e}^{-\tau}}\right\rangle \\
& \times\left(\frac{\Omega_{\mathrm{a}}}{\Omega_{\mathrm{s}}}\right) \int T_{\mathrm{mb}} \mathrm{d} v .
\end{aligned}
$$

This formula is similar to that of Blake et al. (1987) and Turner (1991) but with the introduction of the escape probability and beam dilution factor.

For linear molecules the line strength is equal to the rotational quantum number $J$. The rotational energy level structure 

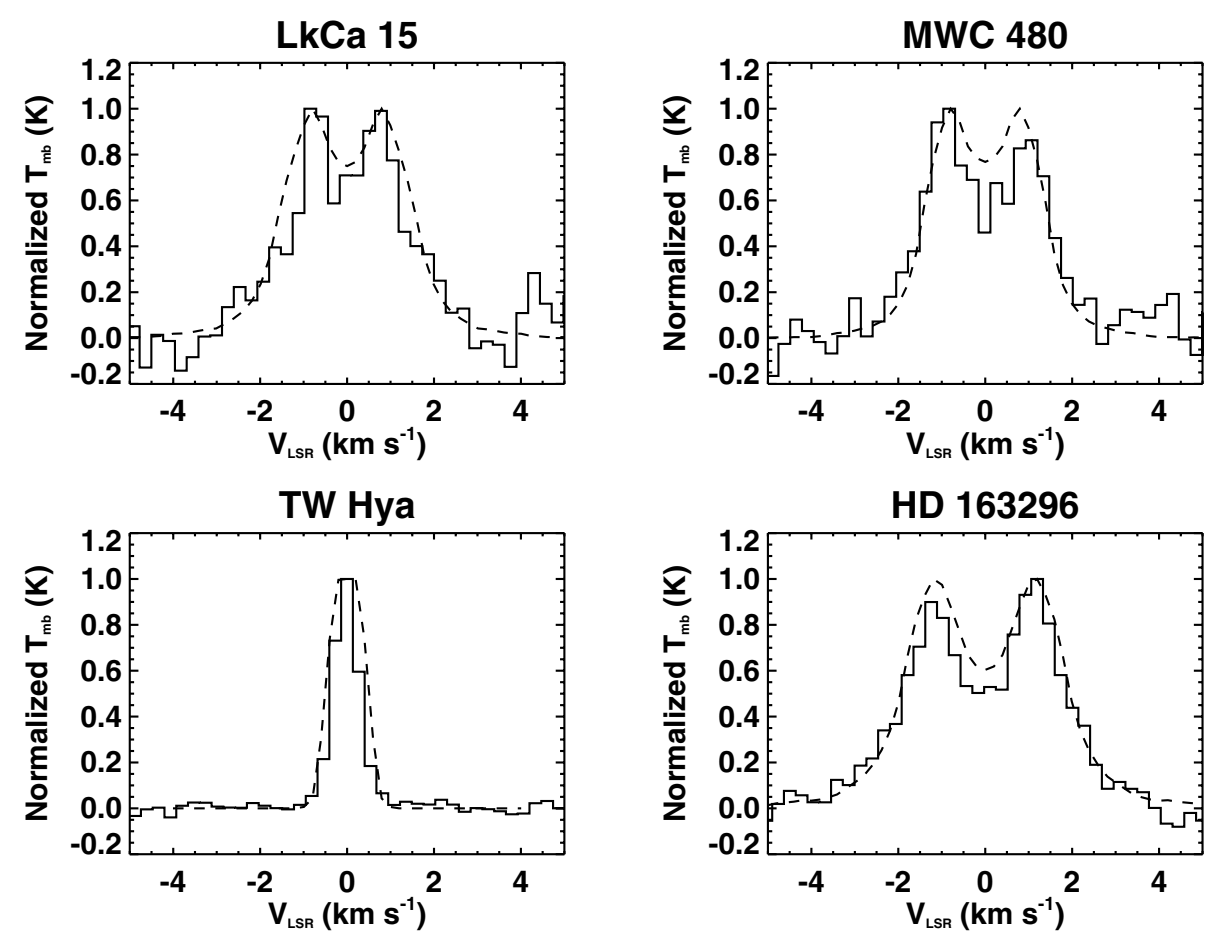

Fig. 6. Observed (full lines) and simulated (dahes lines) ${ }^{12} \mathrm{CO} J=3 \rightarrow 2$ spectra. The observations are normalized to the peak values. The simulations are for a single temperature profile disk model (see Table 7 for parameters).

Table 7. Disk sizes from the ${ }^{12} \mathrm{CO} J=3 \rightarrow 2$ integrated intensities.

\begin{tabular}{llllllll}
\hline \hline & & \multicolumn{3}{c}{ Radius (AU) } & \multicolumn{2}{c}{ Disk model } \\
& Literature $^{a}$ & $T_{\text {ex }}=25 \mathrm{~K}$ & $T_{\text {ex }}=30 \mathrm{~K}$ & $T_{\text {ex }}=50 \mathrm{~K}$ & $i$ & $R_{\text {out }}^{c}$ & $T(1 \mathrm{AU})^{b}$ \\
\hline LkCa 15 $^{\prime}$ & 425 & 620 & 559 & 422 & 57 & 290 & 170 \\
LkCa $15^{d}$ & 425 & $\ldots$ & $\ldots$ & $\ldots$ & 57 & 450 & 100 \\
TW Hya & 200 & 238 & 215 & 162 & 3.5 & 165 & 140 \\
HD 163296 & 310 & 778 & 701 & 530 & 65 & 680 & 180 \\
MWC 480 & 695 & 722 & 650 & 492 & 30 & 400 & 170 \\
\hline
\end{tabular}

${ }^{a}$ See Table 2 for references.

${ }^{b}$ Gas temperature at $1 \mathrm{AU}$. The radius of disk is taken to be $0.01 \mathrm{AU}$.

${ }^{c}$ Values adopted in subsequent analysis.

${ }^{d}$ Alternative fit to LkCa15 data.

of the two linear molecules $\mathrm{CN}$ and $\mathrm{HCN}$ are more complicated than those for $\mathrm{CO}$. The spin of the unpaired electron for $\mathrm{CN}(S=1 / 2)$ and the nuclear spin of ${ }^{14} \mathrm{~N}(I=1)$ lead to fine- and hyperfine splitting of the rotational levels. The observed CN $340 \mathrm{GHz}$ line is a blend of three lines that account for $\simeq 55 \%$ of all the hyperfine lines arising from the level $J=3$ (Simon 1997). The line strength is taken from Avery et al. (1992). An advantage of the hyperfine splitting is that it decreases the optical depth in each individual component. The HCN $J=4 \rightarrow 3$ line is also a blend of hyperfine lines but we assume that all the flux is included in the observed line. Other constants used to derive the column densities are taken from existing catalogs (Pickett et al. 1998) and are summarized in Thi (2002a). The rotational partition functions were calculated using the formulae for each molecule in Gordy \& Cook (1984).

The radical $\mathrm{CN}$ and the molecule $\mathrm{HCN}$ have different critical densities and the HCN $J=4 \rightarrow 3$ line may be subthermally excited in the upper layer, so that the inferred $N(\mathrm{CN}) / N(\mathrm{HCN})$ ratio varies strongly with density. This effect, which can be up to a factor of 2 in the $\mathrm{CN} / \mathrm{HCN}$ abundance ratio, has been corrected using the statistical equilibrium calculations described above for the inferred range of temperatures and densities. It should be noted that this correction assumes that the $\mathrm{CN}$ and HCN lines come from the same location inside the disks, which is probably not the case. In disk models, $\mathrm{CN}$ peaks more toward the lower density surface layers than $\mathrm{HCN}$ because $\mathrm{CN}$ is mostly formed by radical reactions and photodissociation of HCN (Aikawa et al. 2002). This effect would lead to higher $\mathrm{CN} / \mathrm{HCN}$ abundance ratios than presented here.

Table 9 summarizes the beam-averaged column densities and upper-limits for the observed molecules, adopting the disk sizes derived from the fits to the ${ }^{12} \mathrm{CO} J=3 \rightarrow 2$ spectra using the isothermal disk model (see parameters in rightmost columns of Table 7). A single excitation temperature $T_{\text {ex }}=T_{\text {kin }}=25 \mathrm{~K}$ and an optical depth of $\tau=1$ are assumed for all lines. For optically thick lines with $\tau \gg 1$, such as those of 
Table 8. Optical depths estimates from line ratios between isotopologues.

\begin{tabular}{lcccc}
\hline \hline & LkCa15 & TW Hya & HD 163296 & MWC 480 \\
\hline${ }^{12} \mathrm{CO} J=3 \rightarrow 2$ & 26.8 & 8.3 & 18.9 & 14.9 \\
${ }^{13} \mathrm{CO} J=3 \rightarrow 2$ & 0.44 & 0.14 & 0.31 & 0.25 \\
$\mathrm{HCO}^{+} J=4 \rightarrow 3$ & 19.4 & 3.8 & $\ldots$ & $\ldots$ \\
$\mathrm{H}^{13} \mathrm{CO}^{+} J=4 \rightarrow 3$ & $<0.32$ & 0.06 & $\ldots$ & $\ldots$ \\
$\mathrm{HCN} J=4 \rightarrow 3^{13} \mathrm{CN} J=4 \rightarrow 3$ & 0.75 & $<5.1$ & $\ldots$ & $\ldots$ \\
$\mathrm{H}^{13} \mathrm{C}=12$ & $<0.085$ & $\ldots$ & $\ldots$ \\
\hline
\end{tabular}

Note. We assume that the isotopologues have the same excitation temperature and that $\left[{ }^{12} \mathrm{C}\right] /\left[{ }^{13} \mathrm{C}\right]=60$.

$\mathrm{HCO}^{+}$and perhaps $\mathrm{HCN}$, the derived column densities by these formulae are clearly lower limits. Wherever available, column densities derived from isotopic data have been used in these cases.

\subsection{Molecular abundances}

It is well known that disk masses derived from $\mathrm{CO}$ measurements are much lower than those obtained from millimeter continuum observations assuming a gas/dust ratio of 100. Because $\mathrm{CO}$ is subject to photodissociation and freeze-out, one cannot adopt the canonical $\mathrm{CO}$ abundance of $\mathrm{CO} / \mathrm{H}_{2}=10^{-4}$ found for molecular clouds; instead, the disk masses $M_{\text {disk }}$ are assumed to be given by the millimeter continuum observations (see Thi et al. 2001, Table 2).

It should noted that, in the optically thin limit, the abundances are independent of the disk size. The derived abundances are summarized in Table 10. As noted above, the abundances derived from the highly optically thick $\mathrm{HCO}^{+}$and $\mathrm{HCN}$ lines are likely to be underestimated by up to an order of magnitude. For molecules that are detected in all four disks $(\mathrm{CN}$, $\mathrm{HCO}^{+}$), the abundances vary significantly from object to object. The non-detection of HCN toward the Herbig Ae stars confirms the low abundances in these cases, although the high critical density of the $\mathrm{HCN} J=4 \rightarrow 3$ line may also play a role. The upper limits are much lower in the case of TW Hya owing to the small distance of this object and its narrow lines.

The derived abundances and depletion factors are roughly consistent with the large range of values given by van Zadelhoff et al. (2001), especially for their colder models. A full comparison between the two studies is difficult since van Zadelhoff et al. performed more detailed radiative transfer modeling with a varying temperature in the vertical direction. Also, they adopted a smaller disk radius of 200 AU compared with our value of $450 \mathrm{AU}$ for $\mathrm{LkCa} 15$. This leads to higher abundances to reproduce the same line flux, at least for optically thick lines.

For comparison, we have also re-derived the abundances in the disk around DM Tau, where many of the same species have been detected by Dutrey et al. (1997). Their tabulated velocity integrated flux densities have been converted to velocity integrated main beam temperatures via the relation:

$$
\int T_{\mathrm{mb}}(\mathrm{K}) \mathrm{d} v=10^{-23} \times \int F(\mathrm{Jy}) \mathrm{d} v \frac{\lambda^{2}(\mathrm{~cm})}{2 k} \Omega_{\mathrm{a}}^{-1}
$$

where $F$ is the flux density in Jansky, $k$ is the Boltzmann constant in erg $\mathrm{K}^{-1}, \lambda$ the wavelength in $\mathrm{cm}, \Omega_{\mathrm{a}}$ is the main-beam solid angle and $\mathrm{d} v$ is in $\mathrm{km} \mathrm{s}^{-1}$. A total disk mass of $0.018 M_{\odot}$ has been used, computed using Eq. (6) of Thi et al. (2001) and a continuum flux of $110 \mathrm{mJy}$ at $1.3 \mathrm{~mm}$ (Guilloteau \& Dutrey 1998). The abundances are reported in Table 10. Our new abundances estimates are within a factor of 4 of those deduced by Dutrey et al. (1997) who used a different method to derive their abundances.

The last column of Table 10 contains the abundances found in the cold outer region of the protostellar envelope of IRAS 16293-2422. The latter abundances seem to be higher than those in disks by at least an order of magnitude, even taking into account the fact that the disk abundances may be underestimated because of optical depth effects. A noticeable exception is $\mathrm{CN}$, which has a higher abundance in all four disks and in DM Tau than in IRAS 16293-2422.

\section{Discussion}

\subsection{Molecular depletion}

Two processes have been put forward to explain the low molecular abundances in disks. First, in the disk mid-plane, the dust temperature is so low $(<20 \mathrm{~K})$ and the density so high $\left(n_{\mathrm{H}}>10^{9} \mathrm{~cm}^{-3}\right)$ that most molecules including $\mathrm{CO}$ are frozen onto the grain surfaces. This possibility is supported by the detection of large amounts of solid CO at infrared wavelengths in the disk around the younger class I object CRBR 2422.8-3423 (Thi et al. 2002b). In this environment, surface chemistry can occur but the newly-formed species stay in the solid phase and thus remain unobservable at millimeter wavelengths, except for a small fraction which may be removed back in the gas phase by non-thermal desorption processes such as cosmic-ray spot heating.

Second, the photodissociation of molecules in the upper layers of protoplanetary disks by the ultraviolet radiation from the central star and from the ambient interstellar medium can limit the lifetime of molecules. The ultraviolet flux from the central star can reach $10^{4}$ times the interstellar flux (Glassgold et al. 2000). Aikawa et al. (2002) and van Zadelhoff et al. (2003) have modeled the chemistry in disks, taking these mechanisms into account. Their models show that molecules are abundant in the intermediate height regions of disks, consistent with the derived temperature range $(20-40 \mathrm{~K})$ for the emitting gas. According to the flaring disk model, this intermediate region is located just below the warm upper layer $(T \simeq 100 \mathrm{~K})$.

The molecular abundance distributions predicted by the above chemical models including photodissociation and freeze-out have been put into a $2 \mathrm{D}$ radiative transfer code to compute the level populations using statistical equilibrium rather than LTE and to take the optical depth effects properly into account. The resulting integrated fluxes can be compared 
Table 9. Beam averaged column density of various molecules using the disk sizes listed in Table 7.

\begin{tabular}{|c|c|c|c|c|c|}
\hline & & \multicolumn{4}{|c|}{$\bar{N} N\left(\mathrm{~cm}^{-2}\right)$} \\
\hline & & LkCa15 & TW Hya & HD 163296 & MWC 480 \\
\hline Species & Transition & $R_{\text {disk }}=450 \mathrm{AU}$ & $R_{\text {disk }}=165 \mathrm{AU}$ & $R_{\text {disk }}=680 \mathrm{AU}$ & $R_{\text {disk }}=400 \mathrm{AU}$ \\
\hline${ }^{12} \mathrm{CO}^{a}$ & ${ }^{13} \mathrm{CO} J=3 \rightarrow 2$ & $1.9(16)$ & $3.2(16)$ & $3.5(16)$ & $6.9(16)$ \\
\hline${ }^{13} \mathrm{CO}$ & $J=3 \rightarrow 2$ & $3.6(14)$ & $5.5(14)$ & $5.9(14)$ & $1.2(15)$ \\
\hline $\mathrm{HCO}^{+}$ & $J=4 \rightarrow 3$ & $3.3(11)$ & $4.4(12)$ & $9.4(11)$ & $1.0(12)$ \\
\hline $\mathrm{HCO}^{+}$ & $\mathrm{H}^{13} \mathrm{CO}^{+} J=4 \rightarrow 3$ & $\ldots$ & $1.2(13)$ & $\ldots$ & $\ldots$ \\
\hline $\mathrm{H}^{13} \mathrm{CO}^{+}$ & $J=4 \rightarrow 3$ & $<1.5(11)$ & $2.0(11)$ & $\ldots$ & $\ldots$ \\
\hline $\mathrm{DCO}^{+}$ & $J=5 \rightarrow 4$ & $<2.9(11)$ & $4.4(11)$ & $\ldots$ & $\ldots$ \\
\hline $\mathrm{CN}$ & $J=3 \frac{7}{2} \rightarrow 2 \frac{5}{2}$ & $1.5(13)$ & $6.6(13)$ & $1.5(13)$ & $1.5(13)$ \\
\hline $\mathrm{HCN}$ & $J=4 \rightarrow 3$ & $1.8(12)$ & $9.2(12)$ & $<1.0(12)$ & $<1.2(12)$ \\
\hline $\mathrm{H}^{13} \mathrm{CN}$ & $J=4 \rightarrow 3$ & $\ldots$ & $<4.8(11)$ & $\ldots$ & $\ldots$ \\
\hline HNC & $J=4 \rightarrow 3$ & $\ldots$ & $<1.4(12)$ & $\ldots$ & $\ldots$ \\
\hline $\mathrm{DCN}$ & $J=5 \rightarrow 4$ & $\ldots$ & $<4.0(10)$ & $\ldots$ & $\ldots$ \\
\hline CS & $J=7 \rightarrow 6$ & $5.1(12)$ & $\ldots$ & $\ldots$ & $\ldots$ \\
\hline $\mathrm{H}_{2} \mathrm{CO}$ & $J=2_{12} \rightarrow 1_{11}$ & $5.1(12)$ & $\ldots$ & $<2.1(12)$ & $<2.7(13)$ \\
\hline $\mathrm{H}_{2} \mathrm{CO}$ & $J=3_{03} \rightarrow 2_{02}$ & $1.7(12)$ & $\ldots$ & $<2.6(12)$ & $\ldots$ \\
\hline $\mathrm{H}_{2} \mathrm{CO}$ & $J=3_{22} \rightarrow 2_{21}$ & $<1.4(13)$ & $\ldots$ & $<9.4(12)$ & $\ldots$ \\
\hline $\mathrm{H}_{2} \mathrm{CO}$ & $J=3_{12} \rightarrow 2_{11}$ & 7.1(11) & $\ldots$ & $<1.5(12)$ & $<9.4(11)$ \\
\hline $\mathrm{H}_{2} \mathrm{CO}$ & $J=5_{15} \rightarrow 4_{14}$ & $2.4(12)$ & $<8.0(11)$ & $<1.1(12)$ & $<1.5(12)$ \\
\hline $\mathrm{CH}_{3} \mathrm{OH}$ & $J=2_{K} \rightarrow 1_{K}$ & $<7.1(13)$ & $\ldots$ & $<2.8(13)$ & $\ldots$ \\
\hline $\mathrm{CH}_{3} \mathrm{OH}$ & $J=4_{2} \rightarrow 3_{1} \mathrm{E}^{+}$ & $<4.3(14)$ & $\ldots$ & $\ldots$ & $<6.9(13)$ \\
\hline $\mathrm{CH}_{3} \mathrm{OH}$ & $J=5_{K} \rightarrow 4_{K}$ & $<2.4(13)$ & $\ldots$ & $\ldots$ & $\ldots$ \\
\hline $\mathrm{CH}_{3} \mathrm{OH}$ & $J=7_{K} \rightarrow 6_{K}$ & $\ldots$ & $<1.1(13)$ & $\ldots$ & $\ldots$ \\
\hline $\mathrm{N}_{2} \mathrm{H}^{+}$ & $J=4 \rightarrow 3$ & $<1.4(12)$ & $<1.0(13)$ & $\ldots$ & $<1.5(12)$ \\
\hline $\mathrm{H}_{2} \mathrm{D}^{+}$ & $J=1_{10} \rightarrow 1_{11}$ & $<8.7(11)$ & $<4.4(12)$ & $\cdots$ & $<1.0(12)$ \\
\hline SO & $J=8_{8} \rightarrow 7_{7}$ & $\ldots$ & $<4.3(12)$ & $\ldots$ & $\ldots$ \\
\hline
\end{tabular}

Note. The excitation temperature is assumed to be $25 \mathrm{~K}$ for all lines; uncertainties in the column densities are of the order of $30 \%$, not including uncertainties in the disk size.

$a(b)$ means $a \times 10^{b}$.

${ }^{a}{ }^{12} \mathrm{CO}$ column density derived from ${ }^{13} \mathrm{CO}$ intensity assuming $\left[{ }^{12} \mathrm{C}\right] /\left[{ }^{13} \mathrm{C}\right]=60$.

directly with observations. As shown by Aikawa et al. (2002) they differ by factors of a few up to an order of magnitude, which indicates that such models are to first order consistent with the data.

\section{2. $\mathrm{CN} / \mathrm{HCN}$ abundance ratio}

Table 11 includes the $\mathrm{CN} / \mathrm{HCN}$ abundance ratios derived for the disks. Compared with IRAS 16293-2422, the CN/HCN ratio is more than two orders of magnitude larger, and even compared with galactic PDRs, all disk ratios are higher. The disk ratios may be overestimated due to underestimate of $\mathrm{HCN}$ optical depth effects, but the high values are a strong indication that photodissociation processes play a role in the upper layers of the disks.

$\mathrm{CN}$ is particularly enhanced by photochemistry since it is produced by radical reactions involving atomic $\mathrm{C}$ and $\mathrm{N}$ in the upper layers as well as photodissociation of $\mathrm{HCN}$. Moreover, $\mathrm{CN}$ cannot be easily photodissociated itself since very high energy photons $(<1000 \AA,>12.4 \mathrm{eV})$ are required to destroy the radical (van Dishoeck 1987). The $\mathrm{CN} / \mathrm{HCN}$ ratio appears to be higher in disks around Herbig Ae stars than around T Tauri stars, although our high ratio of $>11$ for MWC 480 disagrees with the ratio of $\sim 4$ by Qi (2001) with OVRO. The disagreement between the results of Qi (2001) and ours can be ascribed to the fact that Qi (2001) had $\mathrm{H}^{13} \mathrm{CN}$ data available to constrain the optical depth of the HCN line. Also, their $1-0$ lines are less sensitive to the adopted disk density structure. In general the fluxes from MWC 480 for transitions which have high critical densities are lower than those for T Tauri stars, whereas the $\mathrm{CO}$ fluxes (with lower critical densities) are higher. This may imply that the level populations are subthermal for the disks around Herbig Ae stars.

Van Zadelhoff et al. (2003) have investigated the effects of different UV radiation fields on the disk chemistry, focusing on T Tauri stars with and without excess UV emission. $\mathrm{CN}$ is clearly enhanced in the upper disk layers for radiation fields without any excess UV emission owing to its reduced 
Table 10. Beam-averaged molecular abundances with respect to $\mathrm{H}_{2}$ for the adopted disk sizes.

\begin{tabular}{|c|c|c|c|c|c|c|c|}
\hline \multirow{3}{*}{ Species } & \multicolumn{7}{|c|}{$X=N / N\left(\mathrm{H}_{2}\right)$} \\
\hline & \multirow[t]{2}{*}{$\mathrm{LkCa} 15$} & \multirow[t]{2}{*}{ TW Hya } & \multirow[t]{2}{*}{ HD 163296} & \multirow[t]{2}{*}{ MWC 480} & \multicolumn{2}{|c|}{ DM Tau } & \multirow[t]{2}{*}{ IRAS $16293-2422^{b}$} \\
\hline & & & & & This work $^{a}$ & Dutrey et al. & \\
\hline $\mathrm{CO}$ & $3.4(-07)$ & $5.7(-08)$ & $3.1(-07)$ & $6.9(-07)$ & $9.6(-06)$ & $1.5(-05)$ & $4.0(-05)$ \\
\hline $\mathrm{HCO}^{+}$ & $5.6(-12)$ & $2.2(-11)^{c}$ & $7.8(-12)$ & $1.0(-10)$ & $7.4(-10)$ & $7.4(-10)$ & $1.4(-09)$ \\
\hline $\mathrm{H}^{13} \mathrm{CO}^{+}$ & $<2.6(-12)$ & $3.6(-13)$ & $\ldots$ & $\ldots$ & $<3.6(-11)$ & $<3.6(-11)$ & $2.4(-11)$ \\
\hline $\mathrm{DCO}^{+}$ & $<2.31(-12)$ & $7.8(-13)$ & $\ldots$ & $\ldots$ & $\ldots$ & $\ldots$ & $1.3(-11)$ \\
\hline $\mathrm{CN}$ & $2.4(-10)$ & $1.2(-10)$ & $1.3(-10)$ & $1.4(-10)$ & $9.0(-09)$ & $3.2(-09)$ & $8.0(-11)$ \\
\hline $\mathrm{HCN}$ & $3.1(-11)$ & $1.6(-11)$ & $<9.1(-12)$ & $<1.1(-11)$ & $4.9(-10)$ & $5.5(-10)$ & $1.1(-09)$ \\
\hline $\mathrm{H}^{13} \mathrm{CN}$ & $\ldots$ & $<8.4(-13)$ & $\ldots$ & $\ldots$ & $\ldots$ & $\ldots$ & $1.8(-11)$ \\
\hline HNC & $\ldots$ & $<2.6(-12)$ & $\ldots$ & $\ldots$ & $1.5(-10)$ & $2.4(-10)$ & $6.9(-11)$ \\
\hline $\mathrm{DCN}$ & $\ldots$ & $<7.1(-14)$ & $\ldots$ & $\ldots$ & $\ldots$ & $\ldots$ & $1.3(-11)$ \\
\hline $\mathrm{CS}$ & $<8.5(-11)$ & $\ldots$ & $\ldots$ & $\ldots$ & $2.4(-10)$ & $3.3(-10)$ & $3.0(-09)$ \\
\hline $\mathrm{H}_{2} \mathrm{CO}$ & $4.1(-11)$ & $<1.4(-12)$ & $<1.0(-11)$ & $<1.4(-11)$ & $2.4(-10)$ & $5.0(-10)$ & $7.0(-10)$ \\
\hline $\mathrm{CH}_{3} \mathrm{OH}$ & $<3.7(-10)$ & $<1.9(-11)$ & $<1.5(-10)$ & $<2.0(-09)$ & $\ldots$ & $\ldots$ & $3.5(-10)$ \\
\hline $\mathrm{N}_{2} \mathrm{H}^{+}$ & $<2.3(-11)$ & $<1.8(-11)$ & $\ldots$ & $<1.5(-11)$ & $<5.0(-09)$ & $<2.0(-10)$ & $\ldots$ \\
\hline $\mathrm{H}_{2} \mathrm{D}^{+}$ & $<1.5(-11)$ & $<7.8(-12)$ & $\ldots$ & $<1.0(-11)$ & $\ldots$ & $\ldots$ & $\ldots$ \\
\hline $\mathrm{SO}$ & $\ldots$ & $<4.1(-11)$ & $\ldots$ & $\ldots$ & $\ldots$ & $\ldots$ & $4.4(-09)$ \\
\hline
\end{tabular}

${ }^{a}$ Re-analysis of data from Dutrey et al. (1997), see text.

${ }^{b}$ Outer envelope abundances from Schöier et al. (2002).

${ }^{c}$ Value inferred from $\mathrm{H}^{13} \mathrm{CO}^{+}$.

Table 11. Relative molecular abundances.

\begin{tabular}{lccc}
\hline \hline \multirow{1}{*}{ Source } & $N\left(\mathrm{HCO}^{+}\right)$ & $N(\mathrm{CN})$ & $N\left(\mathrm{DCO}^{+}\right)$ \\
\cline { 2 - 4 } & $N(\mathrm{CO})$ & $N(\mathrm{HCN})$ & $N\left(\mathrm{HCO}^{+}\right)$ \\
\hline LkCa 15 & $1.6(-5)$ & 7.9 & $<0.411$ \\
TW Hya & $3.8(-4)^{1}$ & 7.1 & 0.035 \\
& $1.4(-4)^{2}$ & & \\
DM Tau $^{a}$ & $5.3(-5)$ & 5.8 & \\
DM Tau $^{b}$ & $7.6(-5)$ & 18.4 & \\
\hline HD 163296 & $2.7(-5)$ & $>12.4$ & \\
MWC 480 $^{\text {IRAS 16293-2422 }}$ & $1.5(-5)$ & $>11.7$ & \\
TMC-1 $^{d}$ & $3.6(-5)$ & 0.07 & \\
Orion Bar $^{e}$ & $1.0(-4)$ & 1.5 & \\
IC 63 $^{f}$ & $2.0(-5)$ & 3.8 & \\
\hline
\end{tabular}

References. ${ }^{a}$ Dutrey et al. (1997); ${ }^{b}$ from Table $7 ;^{c}$ Schöier et al. (2002); ${ }^{d}$ Ohishi et al. (1992); ${ }^{e}$ Hogerheijde et al. (1995); ${ }^{f}$ Jansen et al. (1995).

Notes. ${ }^{1}$ Value derived from $\mathrm{H}^{13} \mathrm{CO}^{+}$, assuming $\left[{ }^{12} \mathrm{C}\right] /\left[{ }^{13} \mathrm{C}\right]=60$. ${ }^{2}$ Value derived from $\mathrm{HCO}^{+}$.

photodissociation. When convolved with the JCMT beams, however, the differences are difficult to discern: the HCN emission is nearly identical for the different radiation fields, whereas the $\mathrm{CN}$ emission varies by only a factor of a few. Since T Tauri stars like TW Hya have been observed to have excess UV emission (Costa et al. 2000) - probably originating from a hot boundary layer between the accretion disk and the star -, the difference in the $\mathrm{CN} / \mathrm{HCN}$ chemistry with the Herbig Ae stars may be smaller than thought on the basis of just the stellar spectra. Bergin et al. (2003) suggest that strong Ly $\alpha$ emission dominates the photodissociation rather than an enhanced continuum flux. Since CN cannot be photodissociated by Ly $\alpha$ radiation but $\mathrm{HCN}$ can (Bergin et al.2003; van Zadelhoff et al. 2003), the $\mathrm{CN} / \mathrm{HCN}$ ratio is naturally enhanced.

Other chemical factors can also affect the $\mathrm{CN} / \mathrm{HCN}$ ratio. Radicals such as $\mathrm{CN}$ are mainly destroyed by atomic oxygen in the gas-phase and therefore a lower oxygen abundance can increase the $\mathrm{CN} / \mathrm{HCN}$ ratio. Since atomic oxygen is a major coolant for the gas, a lower abundance will also maintain a higher mean kinetic temperature. Alternatively, the dust temperature could be in the regime that $\mathrm{HCN}$ is frozen out but $\mathrm{CN}$ not because the two molecules have very different desorption energies $\left(E_{\text {des }}(\mathrm{CN})=1510 \mathrm{~K}\right.$ and $E_{\text {des }}(\mathrm{HCN})=4170 \mathrm{~K}$; Aikawa et al. 1997).

Yet an alternative explanation for high $\mathrm{CN}$ abundances is production by X-ray photons emitted from the active atmosphere of T Tauri stars (e.g., Aikawa \& Herbst 1999a; Lepp \& Dalgarno 1996). TW Hya is a particularly strong X-ray emitter, with a measured X-ray flux 10 times higher than the mean $\mathrm{X}$-ray flux observed toward other T Tauri stars (Kastner et al. 2002). In addition, $\mathrm{H}_{2} v=1 \rightarrow 0 \mathrm{~S}(1)$, another diagnostic line of energetic events, has been observed toward this object (Weintraub et al. 2000). TW Hya may however constitute a special case since neither LkCa 15 nor DM Tau seems to show enhanced X-ray emission, yet they have a similar $\mathrm{CN} / \mathrm{HCN}$ ratio. Further observations of molecules in disks around strong $\mathrm{X}$-ray emitting pre-main-sequence stars are warranted to better constrain the contribution of X-rays on the chemistry in disks.

\section{3. $\mathrm{HCO}^{+} / \mathrm{CO}$ abundance ratio}

Table 11 compares the $\mathrm{HCO}^{+} / \mathrm{CO}$ ratios found in the disks with those found in a protostellar region (IRAS 16293-2422), 
a dark cloud (TMC-1) and two galactic photon-dominated regions (PDRs) (Orion Bar and IC 63). Within a factor of two, all values are very similar, except for the TW Hya disk. It should be noted, however, that except for TW Hya, the ratios in disks have been derived from the optically thick $\mathrm{HCO}^{+}$line and may therefore be underestimates. Indeed, the ratio obtained using the main $\mathrm{HCO}^{+}$isotope for TW Hya is closer to that of the other objects. Observations of $\mathrm{H}^{13} \mathrm{CO}^{+}$for all disks are warranted to make definitive conclusions.

$\mathrm{HCO}^{+}$is produced mainly by the gas phase reaction $\mathrm{H}_{3}^{+}+$ $\mathrm{CO} \rightarrow \mathrm{HCO}^{+}+\mathrm{H}_{2}$. Its formation is increased by enhanced ionization (e.g., by X-ray ionization to form $\mathrm{H}_{3}^{+}$in addition to cosmic rays) and by enhanced depletion (which also enhances $\mathrm{H}_{3}^{+}$, see e.g., Rawlings et al. 1992). The fact that all $\mathrm{HCO}^{+}$abundances in disks are higher than those found in normal clouds (after correction for $\mathrm{HCO}^{+}$optical depths) suggests that these processes may play a large role in the intermediate warm disk layer where both molecules are thought to exist. In this context it is interesting to note that TW Hya has the largest depletion of $\mathrm{CO}$ and is also the most active X-ray emitter (see below).

The derived $\mathrm{HCO}^{+}$abundances provide a lower limit to the ionization fraction in disks. The typical values of $10^{-11}-10^{-10}$ are high enough for the magnetorotational instability to occur and thus provide a source of turbulence and mixing in the disk (e.g., Nomura 2002). Ceccarelli et al. (2004) used their $\mathrm{H}_{2} \mathrm{D}^{+}$ observations toward DM Tau and TW Hya to derive an electron abundance of (4-7) $\times 10^{-10}$, assuming that $\mathrm{H}_{3}^{+}$and $\mathrm{H}_{2} \mathrm{D}^{+}$are the most abundant ions. These values refer to the midplane of those disks where the depletion of $\mathrm{CO}$ enhances the $\mathrm{H}_{2} \mathrm{D}^{+} / \mathrm{H}_{3}^{+}$ ratio (see Sect. 5.5), whereas our values apply to the intermediate layer where most of the $\mathrm{HCO}^{+}$emission arises.

\section{4. $\mathrm{H}_{2} \mathrm{CO} / \mathrm{CH}_{3} \mathrm{OH}$}

Lines of $\mathrm{H}_{2} \mathrm{CO}$ have been detected toward only one source, $\mathrm{LkCa} 15 . \mathrm{CH}_{3} \mathrm{OH}$ is not detected in the single-dish observations of any disks, although it is seen in the OVRO interferometer data toward LkCa 15 by Qi (2001), who derived a $\mathrm{CH}_{3} \mathrm{OH}$ column density of $7-20 \times 10^{14} \mathrm{~cm}^{-2}$ compared with our upper limit of $9.4 \times 10^{14} \mathrm{~cm}^{-2}$. Our upper limits for $\mathrm{H}_{2} \mathrm{CO}$ and $\mathrm{CH}_{3} \mathrm{OH}$ are derived from the spectra with the lowest rms Methanol has been detected from the class 0 protostar L1157 by Goldsmith, Langer \& Velusamy (1999), where its emission has been ascribed to a circumstellar disk. However, this object is much younger than those studied here and presumably has a different physical structure and chemical history.

The $\mathrm{H}_{2} \mathrm{CO} / \mathrm{CH}_{3} \mathrm{OH}$ abundance ratio of $>0.15$ for $\mathrm{LkCa} 15$ is consistent with values found for embedded YSOs (see Table 8 of Schöier et al. 2002 for IRAS 16293-2422 and van der Tak et al. 2000 for the case of massive protostars). Heating of the disk, whether by ultraviolet- or X-rays, should lead to strong ice evaporation and thus to enhanced gasphase abundances for grain-surface products. Both $\mathrm{CH}_{3} \mathrm{OH}$ and $\mathrm{H}_{2} \mathrm{CO}$ have been detected in icy mantles, with the $\mathrm{CH}_{3} \mathrm{OH}$ abundance varying strongly from source to source (Dartois et al. 1999; Keane et al. 2001, Pontoppidan et al. 2003). For the few sources for which both species have been seen, the solid
$\mathrm{H}_{2} \mathrm{CO} / \mathrm{CH}_{3} \mathrm{OH}$ ratio varies from $0.1-1$. Thus, the observed ratio in $\mathrm{LkCa} 15$ could be consistent with grain surface formation of both species. Since their absolute abundances are much lower than typical ice mantle abundances of $10^{-6}$ with respect to $\mathrm{H}_{2}$, this indicates that most of the $\mathrm{CH}_{3} \mathrm{OH}$ and $\mathrm{H}_{2} \mathrm{CO}$, if present, is frozen onto grains. Deeper searches for both species in disks are warranted.

Protoplanetary disks are places where comets may form and their volatile composition may provide constraints on their formation models. The $\mathrm{CH}_{3} \mathrm{OH}$ abundance is known to vary significantly between comets. For example, comet C/1999 H1 (Lee) shows a $\mathrm{CO} / \mathrm{CH}_{3} \mathrm{OH}$ ratio around 1 whereas Hale-Bopp and Hyakutake have ratios of 10 and 14 respectively (Biver et al. 2000). Comet Lee probably belongs to the so-called "methanol-rich comets" group (Bockelée-Morvan et al. 1995; Davies et al. 1993). In addition, the measured CO abundance is $\sim 1.8 \pm 0.2 \%$ compared to $\mathrm{H}_{2} \mathrm{O}, 5$ times less than found in HaleBopp. Alternatively, Mumma et al. (2001) propose that Comet Lee has been heated sufficiently after its formation for $\mathrm{CO}$ to evaporate but not $\mathrm{CH}_{3} \mathrm{OH}$, so that $\mathrm{CH}_{3} \mathrm{OH}$ abundance is not enhanced but rather $\mathrm{CO}$ is depleted. Mumma et al. (2001) notice that the $\mathrm{CH}_{3} \mathrm{OH} / \mathrm{H}_{2} \mathrm{O}$ and $\mathrm{CO} / \mathrm{H}_{2} \mathrm{O}$ ratios vary strongly among comets coming from the giant-planets regions. The picture is not complete since $\mathrm{CO}$ can be converted to $\mathrm{CO}_{2}$, whose abundance is high in interstellar ices (e.g., Ehrenfreund \& Charnley 2000 ) but less well known in comets $(10 \%$ in comet $22 \mathrm{P} / \mathrm{Kopff}$, Crovisier et al. 1999).

Long period comets were probably formed in the JupiterSaturn region (around 5-20 AU), whereas our data are only sensitive to distances of more than 50 AU. It would therefore be more relevant to compare the composition of protoplanetary disks to that of Kuiper Belt Objects, which were formed beyond $50 \mathrm{AU}$ in the solar nebula. The chemical composition of Kuiper Belt Objects is not well known (see Jewitt \& Luu 2000), although observations show that comet nuclei and Kuiper Belt Objects have different surface compositions (Luu \& Jewitt 2002; Jewitt 2002). The nature of Centaur objects is better understood. It is believed that Centaur objects were formed beyond $50 \mathrm{AU}$ and recently entered the planetary zone with orbits crossing those of the outer planets. The best studied Centaur object, 5145 Pholus, shows the presence of $\mathrm{CH}_{3} \mathrm{OH}$ although the exact amount is not well constrained (Cruikshank et al. 1998).

\section{5. $\mathrm{D} / \mathrm{H}$ ratio in a circumstellar disk}

The $\mathrm{D} / \mathrm{H}$ ratio in the TW Hya disk of $0.035 \pm 0.01$ has been derived from the $\mathrm{H}^{13} \mathrm{CO}^{+}$and $\mathrm{DCO}^{+}$column density ratios, assuming an isotopic ratio $\left[{ }^{12} \mathrm{C}\right] /\left[{ }^{13} \mathrm{C}\right]$ of 60 (van Dishoeck et al. 2003). Hints of $\mathrm{H}^{13} \mathrm{CN}$ and $\mathrm{DCN}$ features are seen in the TW Hya spectra, but neither of them is definitely detected. Searches for other deuterated species in the LkCa 15 disk, in particular DCN and HDO, are reported by Kessler et al. (2003).

It should be kept in mind that our observations provide only a value of the $\mathrm{D} / \mathrm{H}$ ratio averaged over the entire disk. Models of the $\mathrm{DCO}^{+} / \mathrm{HCO}^{+}$abundance ratio show that it remains rather constant throughout the disk down to a radius of $100 \mathrm{AU}$, 
however (Aikawa et al. 2002). All values are significantly higher than the elemental $[\mathrm{D}] /[\mathrm{H}]$ abundance of $1.5 \times 10^{-5}$ (Pettini \& Bowen 2001; Moos et al. 2002).

Theoretically, the amount of deuterium fractionation in molecules depends on the gas kinetic temperature, which drives the isotopic exchange reactions, and on the cosmic ray ionization rate (Aikawa \& Herbst 1999b). Also, the abundance is enhanced if $\mathrm{CO}$ is significantly depleted onto grains (Brown \& Millar 1989). Thus, the amount of deuterium fractionation can serve as a tracer of the temperature history of the gas. The deuterium fractionation can be further enhanced by grain-surface formation (Tielens 1983), although not for $\mathrm{DCO}^{+} / \mathrm{HCO}^{+}$. Recent chemical models succeed in explaining the high fractionation observed here and in dark cloud cores (Rodgers \& Millar 1996; Roberts \& Millar 2000; Tiné et al. 2000), but only if significant freeze-out is included (Roberts et al. 2002, 2003). Our observed values are also close to those found in disk models which include a realistic 2D temperature and density profile with freeze-out (Aikawa et al. 2002).

Table 2 in van Dishoeck et al. (2003) compares the D/H ratio found in disks to typical values for the $\mathrm{D} / \mathrm{H}$ ratio in different protostellar and cometary environments. The value found in disks is somewhat higher than that in the low-mass protostellar envelope of IRAS 16293-2422, but comparable to that seen in dark cloud cores. $\mathrm{DCO}^{+} / \mathrm{HCO}^{+}$has not been observed in comets, but the $\mathrm{D} / \mathrm{H}$ ratios derived from $\mathrm{DCN} / \mathrm{HCN}$ in pristine material in jets originating from below the comet surface is found to be similar to that seen for $\mathrm{DCO}^{+} / \mathrm{HCO}^{+}$in the TW Hya disk (Blake et al. 1999). Alternatively, Rodgers \& Charnley (2002) propose that the DCN and HCN seen in these cometary jets are the photodestruction products of large organic molecules or dust grains. In either case, the $\mathrm{D} / \mathrm{H}$ ratio of pristine icy material in comets is high. The similarity suggests that either the gas is kept at low temperatures as it is transported from pre-stellar clouds to disks and eventually to comet-forming regions, or, alternatively, that the $\mathrm{DCO}^{+} / \mathrm{HCO}^{+}$ratio has been reset by the chemistry in disks. Comparison with $\mathrm{D} / \mathrm{H}$ ratios of molecules which likely enter the disks as ices are needed to distinguish these scenarios.

\section{Conclusions}

We surveyed low- and high- $J$ transitions of simple organic molecules in two classical T Tauri and two Herbig Ae stars. Analysis of line ratios indicates that the emission comes from a dense $\left(n_{\mathrm{H}}=10^{6}-10^{8} \mathrm{~cm}^{-3}\right)$ and moderately warm region $(T \simeq$ 20-40 K). Detailed fits to the ${ }^{12} \mathrm{CO} 3-2$ emission line profiles provide independent estimates of the sizes of the disks.

Emission from the ion $\mathrm{HCO}^{+}$and the radical $\mathrm{CN}$ are particularly strong, indicating an active gas-phase chemistry in the surface layers of disks which is affected by UV radiation from the central stars. $\mathrm{H}_{2} \mathrm{CO}$ is detected in one source but $\mathrm{CH}_{3} \mathrm{OH}$ is not observed in any object in our sample. In one source (TW Hya) the detection of $\mathrm{DCO}^{+}$allows to constrain the $\mathrm{DCO}^{+} / \mathrm{HCO}^{+}$ratio to $\sim 0.035$, a value that is higher than that found in the envelopes of low-mass protostars but comparable to that observed in cold dark cores, where fractionation due to low temperature chemistry and $\mathrm{CO}$ freeze-out is important.

This work demonstrates that organic chemistry in disks around low- and intermediate-mass pre-main-sequence stars can now be studied observationally. The detection of molecular species in disks is hampered, however, by the small sizes of disks compared with the actual beams of single-dish telescopes. Moreover, because the total amount of material is small (few $\times 10^{-2} M_{\odot}$ ), the observations are limited to the most abundant species. It is likely that the chemistry in more tenuous disks, in which the ultraviolet radiation can penetrate through the entire disk, is different from that for our objects (e.g., Kamp \& Bertoldi 2000; Kamp et al. 2003). Although the outer disks can be resolved by current millimeter interferometers, integration times are too long to do molecular line surveys and the inner tens of AU are still out of reach. The detection of more complex and much less abundant molecules in protoplanetary disks at different stages of evolution awaits the availability of the Atacama Large Millimeter Array (ALMA). Complementary infrared observations of solid-state species along the line of sight of edge-on protoplanetary disks will help to constrain quantitatively the level of depletion in the mid-plane of disks.

Acknowledgements. W.F.T. thanks PPARC for a Postdoctoral grant to UCL. This work was supported by a Spinoza grant from the Netherlands Organization for Scientific Research to EvD and a postdoctoral grant (614.041.005) to WFT. We thank Remo Tilanus, Fred Baas, Michiel Hogerheijde, Kirsten Knudsen-Kraiberg, Annemieke Boonman, and Peter Papadopoulos, who have performed some of the JCMT observations in service; Geoff Blake, Charlie Qi and Jackie Kessler for communicating their OVRO results prior to publication; and Yuri Aikawa for fruitful discussions on disk models. We acknowledge the IRAM staff at Granada for carrying part of the observations in service mode.

\section{References}

Aikawa, Y., \& Herbst, E. 1999a, A\&A, 351, 233

Aikawa, Y., \& Herbst, E. 1999b, ApJ, 526, 314

Aikawa, Y., Umebayashi, T., Nakano, T., \& Miyama, S. M. 1997, ApJ, 486, L51

Aikawa Y., van Zadelhoff, G.-J., van Dishoeck, E. F., \& Herbst, E. 2002, A\&A, 386, 622

Aikawa Y., Momose, M., Thi, W. F., et al. 2003, PASJ, 55, 11

André, Ph., Ward-Thompson, D., \& Barsony, M. 2000, in Protostars \& Planets IV, ed. V. Mannings, A. P. Boss, \& S. S. Russell (Tucson: Univ. Arizona Press), 59

Avery, L. W., Amano, T., Bell, M. B., et al. 1992, ApJS, 83, 363

Beckwith, S. V. W., \& Sargent, A. I. 1993, ApJ, 402, 280

Beckwith, S. V. W., \& Sargent, A. I. 1996, Nature, 383, 139

Beckwith, S. V. W., Sargent, A. I., Chini, R. S., \& Guesten, R. 1990, AJ, 99, 924

Bell, K. R., Cassen, P. M., Klahr, H. H., \& Henning, T. 1997, ApJ, 486, 372

Bergin, A., Calvet, N., D’Alessio, P., \& Herczeg, G. J. 2003, ApJ, 591, L159

Biver, N., Bockelée-Morvan, D., Henry, F., et al. 2000, AJ, 120, 1554

Blake, G. A., Sutton, E. C., Masson, C. R., \& Phillips, T. G. 1987, ApJ, 315, 621

Blake, G. A., Qi, C., Hogerheijde, M. R., Gurwell, M. A., \& Muhleman, D. O. 1999, Nature, 398, 213 
Bockelée-Morvan, D., Brooke, T. Y., \& Crovisier, J., 1995, Icarus, 116,18

Brittain, S. D., Rettig, T. W., Simon, Th., et al. 2003, ApJ, 588, 535

Brown, P. D., \& Millar, T. J. 1989, MNRAS, 237, 661

Calvet, N., D’ Alessio, P., Hartman, L. et al. 2002, ApJ, 568, 1008

Cazaux, S., Tielens, A. G. G. M., Ceccarelli, C., et al. 2003, ApJ, 593, L51

Ceccarelli, C., Loinard, L., Castets, A., et al. 2001, A\&A, 372, 998

Ceccarelli, C., Dominik, C., Lefloch, B., Caselli, P., \& Caux, E. 2004, ApJL, in press

Chiang, E. I., \& Goldreich, P. 1997, ApJ, 490, 368

Chiang, E. I., Joung, M. K., Creech-Eakman, M. J., et al. 2001, ApJ, 547,1077

Costa, V. M., Lago, M. T. V. T., Norci, L., \& Meurs, E. J. A. 2000, A\&A, 354, 621

Crovisier, J., Encrenaz, Th., Lellouch, E., et al. 1999, in The Universe as Seen by ISO, ed. P. Cox \& M. F. Kessler, ESA-SP 427 (Noordwijk: ESTEC)

Cruikshank, D. P., Roush, T. L., \& Bartholomew, M. J., et al. 1998, Icarus, 135,389

D’Alessio, P., Cantó, J., Hartmann, L., Calvet, N., \& Lizano S. 1999, ApJ, 511, 896

Dartois, E., Schutte, W. A., Geballe, T. R., et al. 1999, A\&A, 342, L32

Dartois, E., Dutrey, A., \& Guilloteau, S. 2003, A\&A, 399, 773

Davies, J. K., Puxley, P. J., Mumma, M. J., et al. 1993, MNRAS, 265, 1022

Dutrey, A., Guilloteau, S., \& Guélin, M. 1997, A\&A, 317, L55

Duvert, G., Guilloteau, S., Ménard, F., Simon, M., \& Dutrey, A. 2000, A\&A, 355, 165

Ehrenfreund, P., \& Charnley, S. 2000, ARA\&A, 38, 427

Glassgold, A. E., Feigelson, E. D., \& Montmerle, T. 2000, in Protostars \& Planets IV, ed. V. Mannings, A. P. Boss, \& S. S. Russell (Tucson: Univ. Arizona Press), 429

Goldsmith, P. F., Langer, W. D., \& Velusamy, T. 1999, ApJ, 519, L173

Gordy, W., \& Cook, R. L. 1984, Microwave Molecular Spectropscopy (New-York: Wiley-Interscience), 2nd edition

Guilloteau, S., \& Dutrey, A. 1998, A\&A, 339, 467

Hogerheijde, M. R., Jansen, D. J., \& van Dishoeck, E. F. 1995, A\&A, 294, 792

Irvine, W. M., Schloerb, F. P., Crovisier, J., Fegley, B. Jr., \& Mumma, M. J. 2000, in Protostars \& Planets IV, ed. V. Mannings, A. P. Boss, \& S. S. Russell (Tucson: Univ. Arizona Press), 1159

Jansen, D. J., van Dishoeck, E. F., \& Black, J. H. 1994, A\&A, 282, 605

Jansen, D. J., van Dishoeck, E. F., Black, J. H., Spaans, M., \& Sosin, C. 1995, A\&A, 302, 223

Jansen, D. J. 1995, Ph.D. Thesis, University of Leiden

Jewitt, D. C., \& Luu, J. 2000, in Protostars \& Planets IV, ed. V. Mannings, A. P. Boss, \& S. S. Russell (Tucson: Univ. Arizona Press), 1201

Jewitt, D. C. 2002, AJ, 123, 1039

Kamp, I., \& Bertoldi, F. 2000, A\&A, 353, 276

Kamp, I., van Zadelhoff, G.-J., van Dishoeck, E. F., \& Stark, R. 2003, A\&A, 397, 1129

Kastner, J. H., Zuckerman, B., Weintraub D. A., \& Forveille T. 1997, Science, 277, 67

Kastner, J. H., Huenemoerder, D. P., Schulz, N. S., Canizares, R. C., \& Weintraub, D. A. 2002, ApJ, 567, 434

Keane, J. V., Tielens, A. G. G. M., Boogert, A. C. A., Schutte, W. A., \& Whittet, D. C. B. 2001, A\&A, 379, 254

Kessler, J. 2003, Ph.D. Thesis, California Institute of Technology

Kessler, J., Blake, G. A., \& Qi, C. 2003, in Chemistry as a diagnostic of star formation, ed. C.L. Curry, \& M. Fich (NRC Press), 188
Kitamura, Y., Momose, M., Yokogawa, S., et al. 2003, ApJ, 581, 357

Krist, J. E., Stapelfeldt, K. R., Ménard, F., Padgett, D. L., \& Burrows, Ch. J. 2000, ApJ, 538, 793

Langer, W. D., van Dishoeck, E. F., Bergin, E. A., et al. 2000, in Protostars \& Planets IV, ed. V. Mannings, A. P. Boss, \& S. S. Russell (Tucson: Univ. Arizona Press), 29

Lepp, S., \& Dalgarno, A. 1996, A\&A, 306, 21

Lissauer, J. 1993, ARA\&A, 31, 129

Luu, J. X., \& Jewiit, D. C. 2002, ARA\&A, 40, 63

Mannings, V., Koerner, D. W., \& Sargent, A. I. 1996, Nature, 388, 555

Mannings, V., \& Sargent, A. I. 1997, ApJ, 490, 792

Moos, H. W., Sembach, K. R., Vidal-Madjar, A., et al. 2002, ApJS, 140,3

Mumma, M. J., McLean, I. S., DiSanti, M. A., et al. 2001, ApJ, 546, 1183

Najita, J., Bergin, E. A., \& Ullom, J. N. 2001, ApJ, 561, 880

Nomura, H. 2002, ApJ, 567, 587

Ohishi, M., Irvine, W. M., \& Kaifu, N. 1992, in Astrochemistry of Cosmic Phenomena, ed. P. D. Singh (Dordrecht: Reidel), IAU Symp., 150, 171

Papadopoulos P. P., \& Seaquist, E. R. 1998, ApJ, 492, 521

Peng, R. S., \& Whiteoak J. B. 1993, MNRAS, 260, 529

Pettini, M., \& Bowen, D. V. 2001, ApJ, 560, 41

Pickett, H. M., Poynter, R. L., Cohen, E. A., et al. 1998, J. Quant. Spectrosc. \& Rad. Transfer 60, 883

Pontoppidan, K. M., Fraser, H. J., Dartois, E., et al. 2003, A\&A, 408, 981

Press W. H., Teukilsky, S. A., Vetterling, W. T., \& Flannery, B. P. 1997, Numerical Recipes in Fortran 77 (Cambridge University Press), 402

Qi, C. 2001, Ph.D. Thesis, California Institute of Technology

Qi, C., Kessler, J. E., Koener, D. W., Sargent, A. I., \& Blake, G. A. 2003, ApJ, 597, 986

Rawlings, J. M. C, Harquist, T. W., Menten, K. M., Williams, D. A. 1992, MNRAS, 255, 471

Roberts, H., \& Millar T. J. 2000, A\&A, 361, 388

Roberts, H., Herbst, E., \& Millar, T. 2002, MNRAS, 336, 283

Roberts, H., Herbst, E., \& Millar, T. 2003, ApJ, 591, L41

Rodgers, S. D., \& Charnley, B. 2002, MNRAS, 330, 660

Rodgers, S. D., \& Millar, T. J. 1996, MNRAS, 280, 1046

Schöier, F. L., Jørgensen, J. K., van Dishoeck, E. F., \& Blake, G. A. 2002, A\&A, 310, 1001

Simon, M., Dutrey, A., \& Guilloteau, S. 2000, ApJ, 545, 1034

Simon, R. 1997, Ph.D. Thesis, Univeristy of Köln

Stern, S. A. 2003, Nature, 424, 639

Thi, W. F. 2002, Ph.D., University of Leiden

Thi, W. F., van Dishoeck, E. F., Blake, G. A., et al. 2001, ApJ, 561, 1074

Thi, W. F., Pontoppidan, K. M., van Dishoeck, E. F., Dartois, E., \& d'Hendecourt, L. 2002, A\&A, 394, L27

Tielens, A. G. G. M. 1983, A\&A, 119, 1777

Tiné, S., Roueff, E., Falgarone, E., Gerin, M., \& Pineau des Forêts, G. 2000, A\&A, 356, 1039

Turner, B. E. 1991, ApJ, 376, 573

van der Tak, F. F. S., van Dishoeck, E. F., \& Caselli, P. 2000, A\&A, 361,327

van Dishoeck, E. F. 1987, in Rate Coefficients in Astrochemistry, ed. T.J. Millar \& D.A. Williams (Dordrecht: Kluwer), 49

van Dishoeck, E. F., Blake, G. A., Jansen, D. J., \& Groesbeck, T. D. 1995, ApJ, 447, 760

van Dishoeck, E. F., \& Blake, G. A. 1998, ARA\&A, 36, 317

van Dishoeck, E. F., Thi, W. F., \& van Zadelhoff, G.-J. 2003, A\&A, 400, L1 
van Zadelhoff, G.-J., van Dishoeck, E. F., Thi, W. F., \& Blake, G. A. 2001, A\&A, 377, 566

van Zadelhoff, G.-J., Aikawa, Y., Hogerheijde, M. R., \& van Dishoeck, E. F. 2003, A\&A, 397, 789

Webb, R. A., Zuckerman, B., Platais, I., et al. 1999, ApJ, 512, 63

Weinberger, A. J., Becklin, E. E., Schneider, G., et al. 2002, ApJ, 566, 409

Weintraub, D. A., Sandell, G., \& Duncan, W. D. 1989, ApJ, 340, L69

Weintraub, D. A., Kastner, J. H., \& Bary, J. S. 2000, ApJ, 541, 767

Westley, M. S., Baragiola, R. A., Johnson, R. E., \& Baratta, G. A. 1995, Nature, 373, 405
Willacy, K., \& Langer, W. D. 2000, ApJ, 544, 903

Wilner, D. J., Ho, P. T. P., Kastner, J. H., \& Rodriguez, L. F. 2000, ApJ, 534, L101

Wilner, D. J., Bourke, T. L., Wright, C. M., et al. 2003, ApJ, 596, 597

Wuchterl, G., Guillot, T., \& Lissauer, J. J. 2000, in Protostars \& Planets IV, ed. V. Mannings, A. P. Boss, \& S. S. Russell (Tucson: Univ. Arizona Press), 1081

Zuckerman, B., Webb, R. A., Schwartz, M., \& Becklin, E. E. 2000, ApJ, 549, 233 
W.-F. Thi et al.: Molecules in protoplanetary disks, Online Material p 1

\section{Online Material}


W.-F. Thi et al.: Molecules in protoplanetary disks, Online Material p 2

Table 3. Antenna temperatures and noise per channel width for the T Tauri stars

\begin{tabular}{|c|c|c|c|c|}
\hline & Telescope & $\begin{array}{c}\mathrm{d} v^{a} \\
\left(\mathrm{~km} \mathrm{~s}^{-1}\right)\end{array}$ & $\begin{array}{l}T_{\mathrm{A}}^{*} \\
(\mathrm{~K}) \\
\end{array}$ & $\begin{array}{c}\delta T_{\text {ther }}^{a} \\
(\mathrm{~K}) \\
\end{array}$ \\
\hline \multicolumn{5}{|c|}{ LkCa 15} \\
\hline${ }^{12} \mathrm{CO} J=2 \rightarrow 1$ & IRAM30 m & 0.10 & 0.402 & 0.159 \\
\hline${ }^{12} \mathrm{CO} J=3 \rightarrow 2$ & JCMT & 0.13 & 0.258 & 0.051 \\
\hline${ }^{13} \mathrm{CO} J=3 \rightarrow 2$ & JCMT & 0.28 & 0.074 & 0.026 \\
\hline $\mathrm{C}^{18} \mathrm{O} J=2 \rightarrow 1$ & JCMT & 0.21 & $<0.084(2 \sigma)$ & 0.042 \\
\hline $\mathrm{C}^{18} \mathrm{O} J=3 \rightarrow 2$ & JCMT & 0.14 & $<0.054(2 \sigma)$ & 0.027 \\
\hline $\mathrm{HCO}^{+} J=4 \rightarrow 3$ & JCMT & 0.26 & 0.055 & 0.018 \\
\hline $\mathrm{H}^{13} \mathrm{CO}^{+} J=4 \rightarrow 3$ & JCMT & 0.13 & $<0.078(2 \sigma)$ & 0.039 \\
\hline $\mathrm{DCO}^{+} J=5 \rightarrow 4$ & JCMT & 0.13 & $<0.048(2 \sigma)$ & 0.024 \\
\hline $\mathrm{CN} J=3 \frac{7}{2} \rightarrow 2 \frac{5}{2}$ & JCMT & 0.27 & 0.070 & 0.018 \\
\hline $\mathrm{HCN} J=4 \rightarrow 3$ & JCMT & 0.26 & 0.051 & 0.022 \\
\hline $\mathrm{CS} J=7 \rightarrow 6$ & JCMT & 0.27 & $<0.038(2 \sigma)$ & 0.019 \\
\hline $\mathrm{H}_{2} \mathrm{CO} J=2_{12} \rightarrow 1_{11}$ & IRAM30 m & 0.16 & 0.021 & 0.008 \\
\hline $\mathrm{H}_{2} \mathrm{CO} \mathrm{J}=3_{03} \rightarrow 2_{02}$ & IRAM30 m & 0.10 & 0.025 & 0.021 \\
\hline $\mathrm{H}_{2} \mathrm{CO} \mathrm{J}=3_{22} \rightarrow 2_{21}$ & IRAM30 m & 0.10 & $<0.036(2 \sigma)$ & 0.018 \\
\hline $\mathrm{H}_{2} \mathrm{CO} J=3_{12} \rightarrow 2_{11}$ & IRAM30 m & 0.10 & 0.066 & 0.016 \\
\hline $\mathrm{H}_{2} \mathrm{CO} J=5_{15} \rightarrow 4_{14}$ & JCMT & 0.27 & 0.030 & 0.016 \\
\hline $\mathrm{CH}_{3} \mathrm{OH} J=2_{K} \rightarrow 1_{K}$ & IRAM30 m & 0.12 & $<0.014(2 \sigma)$ & 0.007 \\
\hline $\mathrm{CH}_{3} \mathrm{OH} J=4_{2} \rightarrow 3_{1} \mathrm{E}^{+}$ & IRAM30 m & 0.11 & $<0.036(2 \sigma)$ & 0.018 \\
\hline $\mathrm{CH}_{3} \mathrm{OH} J=5_{K} \rightarrow 4_{K}$ & IRAM30 m & 0.09 & $<0.036(2 \sigma)$ & 0.018 \\
\hline $\mathrm{N}_{2} \mathrm{H}^{+} J=4 \rightarrow 3$ & JCMT & 0.12 & $<0.454(2 \sigma)$ & 0.227 \\
\hline $\mathrm{H}_{2} \mathrm{D}^{+} J=1_{10} \rightarrow 1_{11}$ & JCMT & 0.12 & $<0.372(2 \sigma)$ & 0.186 \\
\hline \multicolumn{5}{|c|}{ TW Hya } \\
\hline${ }^{12} \mathrm{CO} \mathrm{J}=3 \rightarrow 2^{b}$ & JCMT & 0.13 & 1.853 & 0.066 \\
\hline${ }^{12} \mathrm{CO} \mathrm{J}=3 \rightarrow 2^{c}$ & JCMT & 0.13 & 1.679 & 0.241 \\
\hline${ }^{13} \mathrm{CO} J=3 \rightarrow 2$ & JCMT & 0.14 & 0.185 & 0.046 \\
\hline $\mathrm{HCO}^{+} J=4 \rightarrow 3^{b}$ & JCMT & 0.13 & 0.656 & 0.230 \\
\hline $\mathrm{HCO}^{+} J=4 \rightarrow 3^{c}$ & JCMT & 0.13 & 1.197 & 0.087 \\
\hline $\mathrm{H}^{13} \mathrm{CO}^{+} J=4 \rightarrow 3$ & JCMT & 0.13 & 0.050 & 0.016 \\
\hline $\mathrm{DCO}^{+} J=5 \rightarrow 4$ & JCMT & 0.13 & 0.104 & 0.023 \\
\hline $\mathrm{CN} J=3 \frac{7}{2} \rightarrow 2 \frac{5}{2}$ & JCMT & 0.14 & 0.597 & 0.075 \\
\hline $\mathrm{HCN} J=4 \rightarrow 3$ & JCMT & 0.13 & 0.369 & 0.080 \\
\hline $\mathrm{H}^{13} \mathrm{CN} \mathrm{J}=4 \rightarrow 3$ & JCMT & 0.13 & $<0.056(2 \sigma)$ & 0.028 \\
\hline $\mathrm{HNC} J=4 \rightarrow 3$ & JCMT & 0.13 & $<0.089(2 \sigma)$ & 0.049 \\
\hline $\mathrm{DCN} J=5 \rightarrow 4$ & JCMT & 0.13 & $<0.068(2 \sigma)$ & 0.034 \\
\hline $\mathrm{H}_{2} \mathrm{CO} J=3_{12} \rightarrow 2_{11}$ & JCMT & 0.10 & $<0.088(2 \sigma)$ & 0.044 \\
\hline $\mathrm{H}_{2} \mathrm{CO} J=5_{15} \rightarrow 4_{14}$ & JCMT & 0.13 & $<0.074(2 \sigma)$ & 0.037 \\
\hline $\mathrm{CH}_{3} \mathrm{OH} J=7_{K} \rightarrow 6_{K}$ & JCMT & 0.14 & $<0.046(2 \sigma)$ & 0.023 \\
\hline $\mathrm{N}_{2} \mathrm{H}^{+} J=4 \rightarrow 3$ & JCMT & 0.12 & $<0.710(2 \sigma)$ & 0.355 \\
\hline $\mathrm{H}_{2} \mathrm{D}^{+} J=1_{10} \rightarrow 1_{11}$ & JCMT & 0.12 & $<0.596(2 \sigma)$ & 0.298 \\
\hline $\mathrm{SO} J=8_{8} \rightarrow 7_{7}$ & JCMT & 0.13 & $<0.202(2 \sigma)$ & 0.101 \\
\hline
\end{tabular}

${ }^{a} \mathrm{~d} v$ is the spectral resolution of the data in $\mathrm{km} \mathrm{s}^{-1} ; T_{\mathrm{A}}^{*}$ is the antenna temperature (not corrected for beam efficiency) and $\delta T_{\text {ther }}$ is the thermal noise per $\mathrm{d} v$ channel. ${ }^{b}$ Data taken in November 1999. ${ }^{c}$ Data taken in May 2004. 
W.-F. Thi et al.: Molecules in protoplanetary disks, Online Material p 3

Table 4. Same than Table 3 but for the Herbig Ae stars.

\begin{tabular}{|c|c|c|c|c|}
\hline & Telescope & $\begin{array}{c}\mathrm{d} v \\
\left(\mathrm{~km} \mathrm{~s}^{-1}\right)\end{array}$ & $\begin{array}{l}T_{\mathrm{A}}^{*} \\
(\mathrm{~K})\end{array}$ & $\begin{array}{l}T_{\text {ther }} \\
(\mathrm{K})\end{array}$ \\
\hline \multicolumn{5}{|c|}{ HD 163296} \\
\hline${ }^{12} \mathrm{CO} J=3 \rightarrow 2$ & JCMT & 0.23 & 0.920 & 0.130 \\
\hline${ }^{13} \mathrm{CO} J=3 \rightarrow 2$ & JCMT & 0.28 & 0.225 & 0.052 \\
\hline $\mathrm{HCO}^{+} J=4 \rightarrow 3$ & JCMT & 0.26 & 0.128 & 0.030 \\
\hline $\mathrm{CN} J=3 \frac{7}{2} \rightarrow 2 \frac{5}{2}$ & JCMT & 0.27 & 0.115 & 0.018 \\
\hline $\mathrm{HCN} J=4 \rightarrow 3^{2}$ & JCMT & 0.26 & $<0.074(2 \sigma)$ & 0.037 \\
\hline $\mathrm{H}_{2} \mathrm{CO} J=5_{15} \rightarrow 4_{14}$ & JCMT & 0.27 & $<0.204(2 \sigma)$ & 0.102 \\
\hline $\mathrm{H}_{2} \mathrm{CO} J=2_{12} \rightarrow 1_{11}$ & IRAM30 m & 0.17 & $<0.018(2 \sigma)$ & 0.009 \\
\hline $\mathrm{H}_{2} \mathrm{CO} J=3_{03} \rightarrow 2_{02}$ & IRAM30 m & 0.11 & $<0.030(2 \sigma)$ & 0.015 \\
\hline $\mathrm{H}_{2} \mathrm{CO} J=3_{12} \rightarrow 2_{11}$ & IRAM30 m & 0.10 & $<0.018(2 \sigma)$ & 0.009 \\
\hline $\mathrm{CH}_{3} \mathrm{OH} J=2_{K} \rightarrow 1_{K}$ & IRAM30 m & 0.12 & $<0.014(2 \sigma)$ & 0.007 \\
\hline \multicolumn{5}{|c|}{ MWC 480} \\
\hline${ }^{12} \mathrm{CO} J=3 \rightarrow 2$ & JCMT & 0.13 & 0.498 & 0.073 \\
\hline${ }^{13} \mathrm{CO} J=3 \rightarrow 2$ & JCMT & 0.28 & 0.102 & 0.016 \\
\hline $\mathrm{C}^{18} \mathrm{O} J=3 \rightarrow 2$ & JCMT & 0.14 & $<0.062(2 \sigma)$ & 0.031 \\
\hline $\mathrm{HCO}^{+} J=4 \rightarrow 3$ & JCMT & 0.26 & 0.052 & 0.017 \\
\hline $\mathrm{DCO}^{+} J=5 \rightarrow 4$ & JCMT & 0.13 & $<0.106(2 \sigma)$ & 0.053 \\
\hline $\mathrm{CN} J=3 \frac{7}{2} \rightarrow 2 \frac{5}{2}$ & JCMT & 0.27 & 0.038 & 0.017 \\
\hline $\mathrm{CS} J=7 \rightarrow 6$ & JCMT & 0.27 & $<0.040(2 \sigma)$ & 0.020 \\
\hline $\mathrm{HCN} J=4 \rightarrow 3$ & JCMT & 0.53 & $<0.024(2 \sigma)$ & 0.012 \\
\hline $\mathrm{H}_{2} \mathrm{CO} J=3_{12} \rightarrow 2_{11}$ & JCMT & 0.43 & $<0.030(2 \sigma)$ & 0.015 \\
\hline $\mathrm{H}_{2} \mathrm{CO} J=5_{15} \rightarrow 4_{14}$ & JCMT & 0.27 & $<0.032(2 \sigma)$ & 0.016 \\
\hline $\mathrm{H}_{2} \mathrm{D}^{+} J=1_{10} \rightarrow 1_{11}$ & JCMT & 0.12 & $<0.090(2 \sigma)$ & 0.045 \\
\hline $\mathrm{N}_{2} \mathrm{H}^{+} J=4 \rightarrow 3$ & JCMT & 0.12 & $<0.126(2 \sigma)$ & 0.063 \\
\hline $\mathrm{H}_{2} \mathrm{CO} J=2_{12} \rightarrow 1_{11}$ & IRAM30 m & 0.21 & $<0.016(2 \sigma)$ & 0.008 \\
\hline $\mathrm{H}_{2} \mathrm{CO} J=3_{22} \rightarrow 2_{21}$ & IRAM30 m & 1.37 & $<0.006(2 \sigma)$ & 0.003 \\
\hline $\mathrm{CH}_{3} \mathrm{OH} J=4_{2} \rightarrow 3_{1} \mathrm{E}^{+}$ & IRAM30 m & 1.37 & $<0.006(2 \sigma)$ & 0.003 \\
\hline
\end{tabular}


Table 5. Calibration sources used for the JCMT observations.

\begin{tabular}{|c|c|c|c|c|}
\hline Line & $\mathrm{LkCa} 15$ & TW Hya & HD 163296 & MWC 480 \\
\hline${ }^{12} \mathrm{CO} \mathrm{J}=3 \rightarrow 2$ & N2071 IR & $\begin{array}{l}\text { N2071 IR } \\
\text { IRC+10216 }\end{array}$ & $\begin{array}{l}\text { IRC }+10216 \\
\text { IRAS16293-2422 }\end{array}$ & $\mathrm{W} 3(\mathrm{OH})$ \\
\hline${ }^{13} \mathrm{CO} \mathrm{J}=3 \rightarrow 2$ & $\mathrm{~W} 3(\mathrm{OH})$ & IRC +10216 & $\begin{array}{l}\text { NGC6334I } \\
\text { G34.3 } \\
\text { IRAS16293-2422 }\end{array}$ & $\mathrm{W} 3(\mathrm{OH})$ \\
\hline $\mathrm{C}^{18} \mathrm{O} J=2 \rightarrow 1$ & CRL618 & $\ldots$ & $\ldots$ & $\ldots$ \\
\hline $\mathrm{C}^{18} \mathrm{O} J=3 \rightarrow 2$ & $\mathrm{~W} 3(\mathrm{OH})$ & $\ldots$ & $\ldots$ & $\begin{array}{c}\ldots \\
\text { N1333IR4 }\end{array}$ \\
\hline $\mathrm{HCO}^{+} J=4 \rightarrow 3$ & $\mathrm{~W} 3(\mathrm{OH})$ & N2071 IR & G34.3 & $\mathrm{W} 3(\mathrm{OH})$ \\
\hline $\mathrm{H}^{13} \mathrm{CO}^{+} J=4 \rightarrow 3$ & $\mathrm{IRC}+10216$ & $\begin{array}{l}\text { IRC+10216 } \\
\text { N2071 IR }\end{array}$ & ... & $\ldots$ \\
\hline $\mathrm{DCO}^{+} J=5 \rightarrow 4$ & $\begin{array}{l}\text { N1333IR4 } \\
\text { IRC+10216 }\end{array}$ & IRAS16293-2422 & $\ldots$ & $\ldots$ \\
\hline $\mathrm{CN} J=3 \frac{7}{2} \rightarrow 2 \frac{5}{2}$ & CRL618 & CRL618 & $\begin{array}{l}\text { CRL618 } \\
\text { IRAS16293-2422 }\end{array}$ & $\begin{array}{l}\text { CRL618 } \\
\text { G34.3 }\end{array}$ \\
\hline $\mathrm{HCN} J=4 \rightarrow 3$ & CRL618 & IRC +10216 & IRAS16293-2422 & $\begin{array}{l}\text { W3(OH) } \\
\text { CRL618 } \\
\text { OMC1 }\end{array}$ \\
\hline $\mathrm{HNC} J=4 \rightarrow 3$ & $\ldots$ & N2071 IR & $\ldots$ & $\ldots$ \\
\hline $\mathrm{DCN} J=5 \rightarrow 4$ & $\ldots$ & $\begin{array}{l}\text { N2071 IR } \\
\text { IRC+10216 }\end{array}$ & $\ldots$ & $\ldots$ \\
\hline $\mathrm{H}^{13} \mathrm{CN} J=4 \rightarrow 3$ & not available & $\begin{array}{c}\ldots \\
\text { IRAS16293-2422 }\end{array}$ & $\ldots$ & $\ldots$ \\
\hline $\mathrm{CS} J=7 \rightarrow 6$ & not observed & $\ldots$ & $\ldots$ & not observed \\
\hline SO $J=8_{8} \rightarrow 7_{7}$ & $\ldots$ & $\ldots$ & $\ldots$ & $\ldots$ \\
\hline $\mathrm{H}_{2} \mathrm{CO} J=3_{12} \rightarrow 2_{11}$ & $\ldots$ & N2071 IR & $\ldots$ & $\ldots$ \\
\hline $\mathrm{H}_{2} \mathrm{CO} \mathrm{J}=5_{15} \rightarrow 4_{14}$ & CRL618 & $\begin{array}{l}\text { N2071 IR } \\
\text { IRC+10216 }\end{array}$ & IRAS16293-2422 & CRL618 \\
\hline $\mathrm{CH}_{3} \mathrm{OH} J=7_{K} \rightarrow 6_{K}$ & $\ldots$ & not observed & $\ldots$ & $\ldots$ \\
\hline $\mathrm{N}_{2} \mathrm{H}^{+} J=4 \rightarrow 3$ & not available & not available & $\ldots$ & not available \\
\hline $\mathrm{H}_{2} \mathrm{D}^{+} J=1_{10} \rightarrow 1_{11}$ & not available & not available & $\ldots$ & not avaialble \\
\hline
\end{tabular}

Note. When a observation is carried out over more than one run, different standard sources are used depending on their availability. The entry ... indicates that the source was not observed in that particular line (cf. Table 6), so no standard was needed. 